\title{
28th Annual Meeting of the International Society of Blood Purification (ISBP)
}

September 24-26, 2010, Marina del Rey, Calif., USA

\section{Abstracts}

Guest Editors

Victor Gura, Beverly Hills, Calif., USA

Robert Zietse, Rotterdam, The Netherlands

\section{Contents}

Dialysis Strategies

Abstracts 1-6

Dialysates

Abstracts 7-10

Dialysis Technologies

Abstract 11

Monitoring of Dialysis

Abstracts 12-17

Hemodialysis

Abstracts 18-31

Hemodiafiltration

Abstract 32

Complications

Abstracts 33-36

Vascular Access

Abstract 37

New Technologies

Abstracts 38-41

Apheresis

Abstracts 42-43

Author Index 


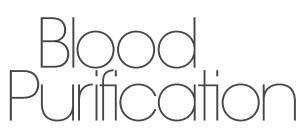

\section{Dialysis Strategies}

1

\section{Fluid Balance During Intestinal Dialysis in a Beluga Whale}

Allison Tuttle ${ }^{1}$, Andre A. Kaplan ${ }^{2}$

${ }^{1}$ Mystic Aquarium, a Division of Sea Research, Inc., Mystic, CT, United States, ${ }^{2}$ Department of Medicine, University of Connectiout Health Center, Farmington, Conn., USA

Background: We have recently reported on our experience in providing renal replacement therapy for a $1050 \mathrm{~kg}$ Beluga whale with AKI (Kaplan \& Tuttle ASN 2010 (abs)). Over a 15 day period, intestinal dialysis was capable of providing control of nitrogen, acid base and potassium balance. BUN decreased from $186 \mathrm{mg} / \mathrm{dL}$ to $136 \mathrm{mg} /$ $\mathrm{dL}, \mathrm{K}$ decreased from $6.9 \mathrm{mEq} / \mathrm{L}$ to $4.1 \mathrm{mEq} / \mathrm{L}$ and $\mathrm{pH}$ rose from 6.99 to 7.38 . We now report on the fluid balance obtained using a solution of $20 \%$ mannitol as a means of increasing solute clearance.

Methods: We originally estimated total body water at 500 liters and were planning on providing a net 100 liters per day of clearance using 30 liters of mannitol and expecting 100 liters of diarrhea fluid to be replaced with parenteral fluids. Despite dividing gastric infusions to 5 times daily, the maximum tolerated mannitol infusion was 10 liters per day. Relying on human experience with mannitol induced diarrhea, we expected intestinal output to contain hyponatric fluid with substantial bicarbonate loss. Given difficulty in obtaining weights and blood pressure, daily fluid infusions were geared to maintain serum sodium. Since acidosis was severe on initiation of dialysis, bicarbonate infusions were chosen to provide a steady increase in serum bicarbonate.

Results: Over the 15 days of treatment a total of 113.5 liter of mannitol was infused by gastric tube. During the same period parenteral infusions included: 35.2 liters of D5W, 216 liters of D5W with $50 \mathrm{mEq}$ of bicarbonate/liter, 94 liters of $\mathrm{NaCl}$ and 108 liters of $\mathrm{NaCl}$ with $50 \mathrm{mEq}$ of bicarbonate/liter. In total, parenteral replacement was 453 liters representing almost exactly 4 times the volume of mannitol ingestion. Serum sodium on the first day of treatment was $148 \mathrm{mEq} / \mathrm{L}$ and was $152 \mathrm{mEq} / \mathrm{L}$ at the end of the treatment. Serum bicarbonate increased from an initial value of $10.3 \mathrm{mEq} / \mathrm{L}$ to $29.4 \mathrm{mEq} / \mathrm{L}$. Despite improvements in nitrogen, potassium and acid base balance, the whale expired on the 15th day. Laboratory values suggest an ongoing hemolytic process which predated the onset of the intestinal dialysis. Although autopsy confirmed ATN, neither the cause of renal failure, nor the cause of death has yet to be ascertained. Nonetheless, autopsy did not show any evidence of pulmonary edema.
Conclusion: We conclude that, despite the ultimate death of the animal, intestinal dialysis appears to be a reasonable option in providing renal replacement therapy in a whale. If intestinal dialysis is employed, intestinal output induced by $20 \%$ mannitol should be expected to be 4 times the ingested volume.

\section{2 \\ Halting the Progression of Chronic Kidney Disease: Experiences with Long Term Total Correction of Anemia}

\author{
Barry von Hartitzsch, A. Deviprakash Udupa, \\ James E. Bourdeau, Jay Fu, Robert M. Gold
}

Nephrology Specialists of Oklahoma, USA

Background: From 1999-2007 using Medicare accepted exceptions for full correction of anemia combined with aggressive treatment of other risk factors we corrected Hemoglobin levels to normal in patients with stage 3 and 4 chronic kidney disease.

Results: With hemoglobin levels in the normal range (12-16 gm/ dl) these patients felt normal, functioned normally, and renal function ceased to progress for periods of 4.5 to 9.5 years. When hemoglobin levels fell into the $10-12 \mathrm{gm} / \mathrm{dl}$ range renal function deteriorated with a rise in serum creatinine reversible if hemoglobin levels were restored quickly to the normal range but permanent if there was a substantial delay in correction. After Sept. 11th 2007 when the FDA required hemoglobin levels in the $10-12 \mathrm{gm} / \mathrm{dl}$ range renal function declined progressively to initiation of dialysis, or death from unresponsive heart failure in less than 2 years. For patients followed a number of years prior to starting erythropoietin at $10 \mathrm{gm} / \mathrm{dl}$ each $1 \mathrm{gm}$ drop in hemoglobin below the normal range for men and post-menopausal women resulted in a $10-15 \mathrm{ml} / \mathrm{min}$ loss of GFR to $20-28 \mathrm{ml} /$ min when most patients start erythropoietin therapy.

Conclusion: We can prevent chronic kidney disease if we start before hypoxic tissue damage occurs with small doses of erythropoietin, when the hemoglobin drops below13.0-13.5 gm/dl in postmenopausal women and $14.0-14.5 \mathrm{gm} / \mathrm{dl}$ in men. Cost $\$ 1500-\$ 2000$ /yr for 30 yrs $\$ 45,000-\$ 60,000$ versus $\$ 80,000$ for 5-6yrs $\$ 400,000$ $\$ 480,000$ for hemodialysis. The cost savings, productivity and quality of life are significantly better.

\begin{tabular}{ll}
\hline KARGER & ( ) 2010 S. Karger AG, Basel \\
0253-5068/10/0303-0222\$26.00/0 \\
$\begin{array}{l}\text { Fax +4161306 1234 } \\
\begin{array}{l}\text { E-Mail karger@karger.ch } \\
\text { www.karger.com }\end{array}\end{array}$ & $\begin{array}{l}\text { Accessible online at: } \\
\text { www.karger.com/bpu }\end{array}$
\end{tabular}




\section{3}

\section{Connecting the Dots--- the Etiology and Pathophysiology of Chronic Kidney Disease}

\section{Barry von Hartitzsch}

Nephrology Specialists of Oklahoma, USA

Background: Chronic hypoxia induces a series of reactions which lead to the replacement of functional renal cells with fibrous tissue: epithelial-to-mesenchymal transition.

Methods: Clinically I have been able to halt the progression of chronic kidney disease under less than ideal conditions for periods of 4.5-9.5 years by recognizing and treating the factors that cause tissue hypoxia. These are anemia (a reduction in oxygen carrying capacity of the blood), smoking, obesity/sleep apnea, hypertension, cardiac disease, and diabetes mellitus.

Results: Each of these conditions has a mechanism, which will be reviewed, by which they individually and collectively reduce oxygen delivery and utilization in the cells creating a failure of intracellular energy production. Cells damaged by hypoxia are phagocytosed by macrophages leading to increased pro inflammatory cytokines TNFa, IL-6, and CRP. This is oxidative stress. The severity of this hypoxic event is measured as the dose of erythropoietin, "erythropoietin resistance", required to raise hemoglobin levels to normal 13.5 $-16 \mathrm{gm} / \mathrm{dl}$ when patients in stage $4 \mathrm{CKD}$ bordering on dialysis will say they "feel normal" again. When the CHOIR trial was critically reviewed by the FDA their analysis shows the highest event and death rates at the lowest achieved hemoglobin levels and the lowest event rates and best survival for 281 patients with achieved hemoglobin levels $>13.3 \mathrm{gm} / \mathrm{dl}$.

Conclusion: There appears to be no rational scientific reason why total correction of anemia along with the other risk factors for chronic kidney disease should not be accomplished to prevent the need for dialysis in most patients.

\section{4 \\ The Manipulation of Data in Clinical Trials: Why Medical Care Should Focus on Patients and Not Statistics}

\author{
Barry von Hartitzsch \\ Nephrology Specialists of Oklahoma, USA
}

Background: Since Medicare began funding ESRD in 1972 regular submission of data on every patient has been required to create a medical data bank for statistical analysis, research, and to guide future treatment. This data shows that when Erythropoietin is used to raise hemoglobin $(\mathrm{Hgb})$ levels and improve tissue oxygen levels blood transfusions and hospitalizations are significantly reduced and survival is best with $\mathrm{Hgb}>13 \mathrm{gm} / \mathrm{dl}$. In the mid 90's Government efforts to curb health care costs brought HMO/PPO expansion, an alliance between Government and Academic Medicine resulting in DOQI guidelines, Clinical Controlled Trials, and an FDA with sweeping powers to control trials, cost, research, and rewrite the "Practice of Medicine".

28th Annual Meeting of the International Society of Blood Purification (ISBP)
Methods: Extensive reviews of the published ESA clinical trials with particular attention to methodology and results were undertaken. I also conducted a complete review of the transcript and all data prepared for, and presented at the FDA Sept. 11 all day meeting at which ESA use in chronic renal failure was lowered to a $10-12 \mathrm{gm} / \mathrm{dl}$ hemoglobin range.

Results: Is it by chance or by design that the high treatment arm of the NHCT, CHOIR, and CREATE trials all have more people with multiple risk factors and severe cardiac disease, who die from anemia with ischemic not thrombotic events as propagated by our non clinical Academic Nephrologists? With the "Clinical Controlled Trial" the FDA has been able to control the data released, control the discourse by limiting all opposing clinical dialogue to just one hour in seven, and achieve their goal with non clinical advisors voting on questions designed to only reduce Hgb levels. FDA data from the NHCT and CHOIR trials which few have seen till now again indicates more events and higher death rates for lower achieved hemoglobin levels with least events and best survival for 281 people with achieved hemoglobin levels $>13 \mathrm{gm} / \mathrm{dl}$. This agrees with the observational data.

Conclusion: At the 10-12 gm/dl range imposed by FDA edicts and CMS reduced payment schedules we are now being used to accelerate the demise of our patients. Our values, our integrity, our profession, and the "TRUST" of our patients are now at risk. We need to act now.

\section{5}

\section{Acidosis in Intensive Care Unit (ICU) Patient's on Continuous Renal Replacement Therapies (CRRT): Classical versus Stewart's Approach}

\author{
Paolo Lentini ${ }^{1}$, Vincenzo Catena ${ }^{2}$, Monica Baccarin ${ }^{2}$, Luca \\ Zanoli3, Alexandra Chronopoulos", Massimo De Cal', \\ Valentina Pellanda ${ }^{1}$, Claudio Ronco ${ }^{4}$, Marco Baiocchi ${ }^{2}$, \\ Roberto Dell'Aquila ${ }^{1}$ \\ ${ }^{1}$ Nephrology, St. Bassiano Hospital, Bassano Del Grappa \\ (VI), Italy, ${ }^{2}$ Intensive Care, St. Bassiano Hospital, Bassano \\ Del Grappa, Italy, ${ }^{3}$ Internal Medicine, University of Catania, \\ Catania, Italy, ${ }^{4}$ Nephrology, St.Bortolo Hospital, Vicenza, \\ Italy
}

Background: Acidosis it's one of the most commonly seen problems in critically ill patients. Respiratory failure, kidney failure, heart failure, Hypoperfusion and intoxications can all result in dangerous shifts of $\mathrm{pH}$ concentration. These disturbances often go unnoticed, especially in the case of combined acid-base disorders. If the perturbations are not recognized and addressed, they may result in poor outcomes in intensive care unit (ICU) patients. Acidosis is one of the most common indications for CRTT in ICU. While common CRRT buffers can provide restauration of internal milieu, with better control of $\mathrm{PH}$ and $\mathrm{HCO}_{3}$, mechanical ventilation (MV) helps control $\mathrm{PaCO}_{2}, \mathrm{PaO}_{2}$ and $\mathrm{O}_{2}$ saturation. The best approach for acid-base analysis is debated. For critically ill patients the Stewart approach may be better than the Classic approach, popularized by Relman and Schwartz, which uses the Bronstead-Lowry definitions and Henderson- Hasselbach and Siggaard-Andersen equations. Aim: Assessment of correlation and agreement between Classical versus 
Stewart approaches for the analysis of acid-base disturbances during CRRT.

Methods: This prospective cohort study took place in a 10 adult medical-surgical Intensive Care Unit (ICU). We enrolled 19 consecutive adult patients on CVVH and mechanical ventilation. All patients were treated with CVVH with a LYNDA ${ }^{\circledR}$ machine, (Bellco, Mirandola, Italy), with a blood flow rate of $250 \mathrm{ml} / \mathrm{min}$ and with a prescribed infusion rate of at the least $35 \mathrm{ml} / \mathrm{Kg} / \mathrm{hr}$. All treatments were performed with polyetersulfone $1.7 \mathrm{~m}^{2}$ dialyzer (DIAPES, Bellco, Mirandola, Italy). We use a standard buffer (5 Liters: Bicarbonate $35 \mathrm{mmol} / \mathrm{L}$, sodium $140 \mathrm{mmol} / \mathrm{L}$, Potassium $2 \mathrm{mmol} / \mathrm{L}$, Calcium 1,75 mmol/L, magnesium $0.5 \mathrm{mml} / \mathrm{L}$, PH 7,4). Demographic and clinical data were collected. The Acute Physiology andChronic Health Evaluation (APACHE) II and Sepsis related Organ Failure Assessment (SOFA) [26] scores were calculated on admission. We calculated [HCO-3] and SBE with the Henderson- Hasselbach and Siggaard-Andersen equations. Based on the SBE, metabolic status was classified as acidosis (SBE -5.0), normal (SBE -4.9 to 4.9) or alkalosis (SBE +5.0). Physicochemical analysis was performed using the Stewart equations modified by Figge et al. The apparent strong ion difference (SIDa) was calculated: $\mathrm{SIDa}=[\mathrm{Na}+]+[\mathrm{K}+]+[\mathrm{Mg}+]+[\mathrm{Ca} 2+]-[\mathrm{Cl}-]-[$ lactate $]$ $(\mathrm{mEq} / \mathrm{L})$. The normal variation for SIDa was defined as $4042 \mathrm{mEq} / \mathrm{L}$. The effective SID (SIDe) was then calculated: SIDe $=1000 \times 2.46 \mathrm{x}$ $10-11 \times \mathrm{PaCO} 2 /(10-\mathrm{pH})+[\mathrm{alb}] \mathrm{x}(0.123 \times \mathrm{pH}-0.631)+[\mathrm{PO} 4-] \times(0.309$ $\mathrm{x} \mathrm{pH}-0.469)(\mathrm{mEq} / \mathrm{L})$. Normal variation for SIDe was defined as 38 $42 \mathrm{mEq} / \mathrm{L}$. Chi square test was used at 0,6,12, and $24 \mathrm{~h}$ after CVVH to compare the prevalence of acidosis detected with $\mathrm{pH}$ vs. SIDe and BE vs. SIDe.

Results: The prevalence of acidosis after CVVH, as assessed by $\mathrm{pH}$ vs. SIDe, was $[36.8 \%$ vs. $94.7 \%(\mathrm{p}<0.001)]$ at $6 \mathrm{~h}, 21.1 \%$ vs. $73.7 \%(\mathrm{p}<0.05)]$ at $12 \mathrm{~h}$, and $[21.1 \%$ vs. $98.6 \%(\mathrm{p}<0.001)]$ at $24 \mathrm{~h}$. The prevalence of acidosis after CVVH, as assessed by SBE vs.SIDe, was $[57.9 \%$ vs. $94.7 \%(\mathrm{p}<0.05)]$ at $6 \mathrm{~h},[63.2 \% \mathrm{vs} .73 .7 \%(\mathrm{p}=\mathrm{NS})]$ at 12 $\mathrm{h}$, and $[63.2 \%$ vs. $98.6 \%(\mathrm{p}<0.05]$ at $24 \mathrm{~h}$.

Conclusion: Stewart approach seems to be more sensitive than Classical approach for detection of acidosis in ICU patients on CRRT.

\section{6}

\section{Kinetics of Beta2-Microglobulin and Urea in High-Efficiency Hemodiafiltration}

\begin{abstract}
Joan Gascó, Victoria Iñigo, Victòria Mascarós, Rosario Bernabeu, Maria Bibiloni, Mar Castillo, Regina Fortuny, Mique/ Servera

Nephrology Department, Son Llàtzer Hospital, Palma, Mallorca, Spain
\end{abstract}

Background: The DOPPS study results suggest that hemodiafiltration (HDF) may improve survival independently of the small molecules dialysis dose. $\beta_{2}$-Microglobulin $\left(\beta_{2} \mathrm{~m}\right)$ has been used as middle molecules marker in convective treatments. A subanalysis of the HEMO study has shown poorer survival of patients in chronic hemodialysis treatment with higher levels of serum $\beta_{2} \mathrm{~m}$ and in patients who were on dialysis $>3.7$ years both $\beta_{2} \mathrm{~m}$ clearance and $\beta_{2} \mathrm{~m}$ $\mathrm{Kt} / \mathrm{V}$ correlated negatively with mortality. Recently a new bicompartimental urea kinetic model (UKM) adapted to different weekly schedules of standard hemodialysis and HDF has been presented. We apply $\beta_{2}$ m kinetics model and this UKM to analyze the results of convective and difusive treatment with high-efficiency HDF in a clinical setting.

Methods: 16 prevalent patients on hemodialysis for 42.2 months $(8 \mathrm{M} / 8 \mathrm{~F}$, mean 52.7 years) started postdilutional on line HDF with dia-

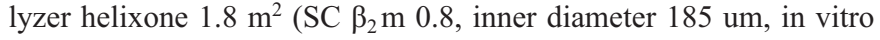
Kuf $59 \mathrm{~mL} / \mathrm{mmHg} / \mathrm{h}$ ), 3 sessions per week of 4 hours, QD $800 \mathrm{ml} /$ min, mean UF $2.1 \mathrm{Kg}$. Monitorized monthly with kinetic model of $\beta_{2} \mathrm{~m}$, and UKM calculated by Solute Solver: $\beta_{2} \mathrm{~m}$ preHDF and $\beta_{2} \mathrm{~m}$ postHDF corrected (by Bergström and Wehle formula), $\% \beta_{2}$ m reduction rate $\left.\beta_{2} \mathrm{~m} \mathrm{mRR}\right) ; \beta_{2} \mathrm{~m}$ clearance $\left(\mathrm{K} \beta_{2} \mathrm{~m}\right)$ by Leypoldt formula, $\beta_{2} \mathrm{~m}$ distribution volume $\left(\beta_{2} \mathrm{mV}\right)$ as $1 / 3$ of the modeled urea single pool volume, $\beta_{2} \mathrm{~m} \mathrm{Kt}$, Kt $\beta_{2} \mathrm{M}$ adjusted to $1.73 \mathrm{~m}^{2}$ of body surface area (SA $\beta_{2} \mathrm{mKt}$ ) and $\beta_{2} \mathrm{M} \mathrm{Kt} / \mathrm{V} ; \mathrm{KoA}$ estimated in vivo (KoA), urea reduction rate (URR), urea diffusive dialyzer clearance (Kd dif), total dialyzer clearance including convective component (Kd), urea distribution volume and generation rate single and double pool (V1p,V2p,G1p,G2p), equilibrated Kt/V (eqKt/V), intercompartmental urea clearance $(\mathrm{Kc})$, normalized protein catabolic rate $(\mathrm{PCRn})$ $1 \mathrm{p}$ and $2 \mathrm{p}$, dialysis standard Kt/V (DstdKt/V), DstdKt/V normalized to SA (SANDstdKt/V). 263 sessions analyzed. Simple and multiple regression analysis with $\beta_{2} \mathrm{mRR}, \mathrm{K} \beta_{2} \mathrm{~m}, \beta_{2} \mathrm{~m} \mathrm{Kt}, \mathrm{SA} \beta_{2} \mathrm{mKt}$ or $\beta_{2} \mathrm{~m}$ $\mathrm{Kt} / \mathrm{V}$ as dependent variables versus other kinetic and clinical variables: effective $\mathrm{Qb}$, convective volume $(\mathrm{CV})$, filtration fraction $(\mathrm{FF})$, hemoglobin preHDF (Hgb), lymphocyte level preHDF (Lymph), albuminemia preHDF(Alb). Plots of kinetic variables categorized by $\mathrm{CV}$ and FF. Data presented as means, 95\% confidence interval and ranges (only means in the abstract).

Results: Mean results of the 263 sessions: effective Qb $323 \mathrm{ml} /$ min, time $242 \mathrm{~min}, \mathrm{CV} 24.09 \mathrm{~L}$, FF 30.7\%, Hgb $12.1 \mathrm{~g} / \mathrm{dL}$, Lymph $1310 \mathrm{cel} / \mathrm{ul}$, Alb $37.5 \mathrm{~g} / \mathrm{L} . \beta_{2} \mathrm{~m}$ pre $21.4, \beta_{2} \mathrm{~m}$ post corrected $4, \beta_{2} \mathrm{~m}$ RR $79.3 \%, \mathrm{~K} \beta_{2} \mathrm{~m} 47.9 \mathrm{ml} / \mathrm{min}, \beta_{2} \mathrm{~m} \mathrm{~V} 9.5 \mathrm{~L}, \beta_{2} \mathrm{~m} \mathrm{Kt} 11.6 \mathrm{~L}$, SA $\beta_{2} \mathrm{mKt} 12.3 \mathrm{~L}, \beta_{2} \mathrm{M} \mathrm{Kt} / \mathrm{V} 1.29$, KoA $722 \mathrm{ml} / \mathrm{min}$, URR 79.6\%, Kd dif $205 \mathrm{ml} / \mathrm{min}, \mathrm{Kd} 228 \mathrm{ml} / \mathrm{min}, \mathrm{V} 1 \mathrm{p} 28.6 \mathrm{~L}, \mathrm{~V} 2 \mathrm{p} 27.1 \mathrm{~L}, \mathrm{G} 1 \mathrm{p} 4.63 \mathrm{mg} /$ min,G2p $4.16 \mathrm{mg} / \mathrm{min}$, eqKt/V 1.75, Kc $434 \mathrm{ml} / \mathrm{min}$, PCRn1p $1.06 \mathrm{~g} /$ $\mathrm{kg} / \mathrm{d}$, PCRn2p $1.16 \mathrm{~g} / \mathrm{kg} / \mathrm{d}$, DstdKt/V 2.76, SANDstdKt/V 2.7. In the multiple regression analysis $\mathrm{CV}$ was the stronger continuous predic-

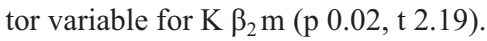

Conclusions: An 25\% increase of CV from 20-22 L to 25-27 $\mathrm{L}$ increases the convective treatment results of high-efficiency HDF, calculated as $\mathrm{K} \beta_{2} \mathrm{~m}$, in a $50 \%$. For an objective of $\beta_{2} \mathrm{M} \mathrm{Kt} / \mathrm{V}$ of 1.5 (rebound near $50 \%$ ) with 4 hours of treatment, a CV of $25 \mathrm{~L}$ is needed. Higher FF produced higher $\mathrm{K} \beta_{2} \mathrm{~m}$ and $\beta_{2} \mathrm{mRR}$ until a $\mathrm{FF}$ of $31 \%$, without better results or declining outcomes over that level. Urea $\mathrm{K}$ dif results lowered with $\mathrm{FF}>25 \%$ but with similar urea $\mathrm{Kd}$. 


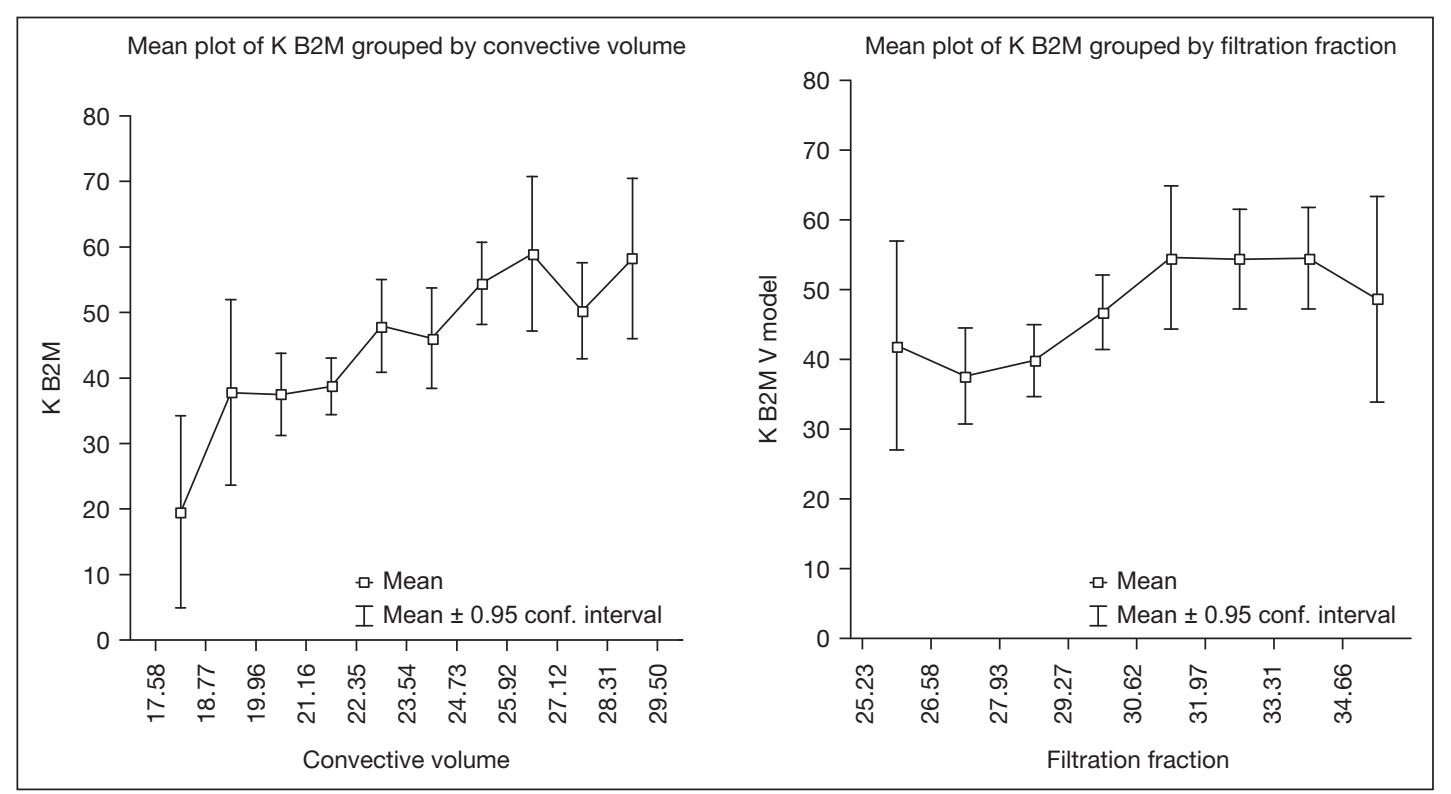

Figure for abstract 6 .

\section{Dialysates}

\section{7}

\section{Combined Extended Haemodialysis with Multiple-Pass Albumin Dialysis (RAED)}

Rosa Diez Guillermo1, Gadano Adrian², Giannasi Sergio3, Greloni Gustavo1, Crucelegui Soledad1, Varela Federico1, Bedini Rocca Mariela1, Serra Diego', San Roman Eduardo ${ }^{2}$, Algranati Salomon ${ }^{1}$

'Department of Nephrology-Hospital Italiano de Buenos Aires, ${ }^{2}$ Department of Hepatology-Hospital Italiano de Buenos Aires, ${ }^{3}$ Department of Critical Care-Hospital Italiano de Buenos Aires, Argentina

Background: Extended hemodialysis (E-HD) plus single-pass albumin dialysis has shown to be a useful option to treat individuals with severe combined renal and liver failure; it is easy to implement but expensive due to the large amount of albumin that has to be used. Aimed at sparing dialysate, dialysate recirculation has also been used in continuous hemodialysis treatments. The recirculation of an albumin-enriched dialysate might allow to save albumin without reducing treatment efficacy. The main aim of this study was to describe a novel technique (RAED) developed by our group.

Methods: Two cases in which this technique was carried out are presented in this report. RAED was carried out during a 6-8 hourperiod through a conventional hemodialysis machine (Fresenius 4008 B) with two high flux polysulphone dialysers (Fresenius HF 60) arranged in-serie. The blood flow was $150 \mathrm{ml} / \mathrm{min}$ whereas the flow of the conventional dialysate circulating through the first dialyzer was set-up at $300 \mathrm{ml} / \mathrm{min}$ and the $5 \%$ albumin dialysate flow through the 2nd filter was of $50 \mathrm{ml} / \mathrm{min}$. This fluid was under continuous recirculation by mean of a double rail peristaltic pump allowing the dialysate to be transferred from a collecting bag to the dialyser and vice versa. A total of $1.5 \mathrm{~L}$ of a $5 \%$ albumin solution was used in each session.

Results: Patient 1: 31 y/o male individual with acute haepatitis and anuric acute renal failure. PHB: Massive necrotizing haepatitis. PRB: billrirubin-associated nephrosis plus acute tubular necrosis. Lab: ALT $130 \mathrm{UI} / \mathrm{L}$, alkaline phosphatase (AP) $82 \mathrm{UI} / \mathrm{L}$, total billirubin: $60 \mathrm{mg} / \mathrm{dL}$, direct BR: $38 \mathrm{mg} / \mathrm{dL}$, serum creatinine (Scr): 7.7 $\mathrm{mg} / \mathrm{dL}$, urea: $154 \mathrm{mg} / \mathrm{dL}$ and Factor V: $60 \mathrm{UI} /$ LPatient 2: 49 y/o male with a past medical history of autoimmune haepatitis and double liver transplant few years ago. CKD with a PRB showing sign of calcineurin inhibitor-associated chronic renal disease; and PHB displaying severe ductopenic rejection. He experienced severe decrease in GFR and was submitted to RRT and included in waiting list for combined liver and kidney transplant. Cholestasis-induced severe pruritus was also reported. Lab results showed: AST: $58 \mathrm{UI} / \mathrm{L}$, ALT: $68 \mathrm{UI} / \mathrm{L}, \mathrm{AP}$ : $470 \mathrm{UI} / \mathrm{L}$, Scr: $4.4 \mathrm{mg} / \mathrm{dL}$, urea: $255 \mathrm{mg} / \mathrm{dL}$, serum albumin: $2.6 \mathrm{mg} /$ $\mathrm{dL}$, total BR: $42 \mathrm{mg} / \mathrm{dL}$, direct BR: $25 \mathrm{mg} / \mathrm{dL}$. Conventional measured failed to improve pruritus. Both individuals underwent E-HD but were rapidly shifted to RAED. The outcome of BR as well as the clinical manifestations were assessed. A significantly larger reduction in serum BR levels was obtained with RAED as compared to that observed with E-HD. This reduction allowed to improve both, renal funcion and pruritus.

Conclusion: Combined E-HD with multiple-pass albumin dialysis is a technique that may have a role in the treatment of combined liver and kidney failure. Although it may not be as efficient as newer therapies, it is less expensive and enables the treatment of ICU patients with hepatorenal failure in developing countries. Additional studies to determine its efficacy are warranted. 


\section{8}

\section{An Examination of Dialysate Quality thru Water Evaluation Method Pressure Load Using the Membrane Filtration Method}

Yutaka Isakozawa, Yoshihiro Tange, Shingo Takesawa, Vee David

Department of Medical Engineering, College of Health

Sciences, Kyushu University of Health and Welfare, Japan

Background: As for the dialysate cleaning criteria, an important point has been put forward in the endotoxin levels, but the bacterial count method is generic as seen globally. As a result having examined the dialysate quality in the water evaluation method, there was no correlation between endotoxin levels and the bacterial count, and endotoxin level was detectably less, it became clear that live germs and gram positive bacteria might be present. The membrane filtration method we are more high sensitive is suitable, and to measure bacterial count in the dialysate. Also, it is thought that an individual difference occurs by the infusion rate of sampling method, and to degree of the pressure in the bacterial count measurement. Therefore we evaluated bacterial count control by the pressure load used for dialysate water quality management in this research.

Methods: Three pressures were set in this study as follows $-100 \mathrm{kPa}$ (depression filtration), $0 \mathrm{kPa}$ (at $1 \mathrm{~atm}$ ) and $+100 \mathrm{kPa}$ (pressurization filtration), and watching the level in the pressure transducer. A bacterial count hypothesized that ten to the eighth $\mathrm{CFU} / \mathrm{mL}$ was present, and the adjustment of the bacteria liquid to become ten to the second CFU/mL (specimen 1), ten to the third CFU/mL (specimen 2). On the membrane filtration kit, we used $37 \mathrm{~mm}$ quality monitor (PALL) which was membrane filter of the cartridge structure with almost no contamination. We distilled each specimen of $100 \mu$ with a $20 \mathrm{~mL}$ of sterilized water and examined depression filtration, the pressure filtration by syringe operation $(\mathrm{N}=3)$ and used $\mathrm{m}$-TGE culture media for culture medium and performed the measurement procedure in all clean bench. $20 \mathrm{~mL}$ of distilled specimen 1 solution contained 10CFU, and distilled specimen 2 solution contained 100CFU.

Results: In the examination of the bacterial culture by the membrane filtration method, bacteria were detected in membrane in itself. As a result of having cultured filtration water after the filtration, the colony was not detected. However, in many dialysis facilities, we must grasp how contamination can be expected because there are few institutions which perform the procedure using clean bench. As a result of having examined the bacterial count change by the membrane filtration method, after the fourth day, it revealed the elevation of the bacterial count, and the change of the bacterial count was not seen after the seventh day. In the results of the bacterial count control by pressure changes, it was with $2-14 \mathrm{CFU} / 20 \mathrm{~mL}$ (average: $8 \mathrm{CFU} / 20 \mathrm{~mL}$ ) with specimen 1 , and $9-71 \mathrm{CFU} / 20 \mathrm{~mL}$ (average: $46 \mathrm{CFU} / 20 \mathrm{~mL}$ ) with specimen 2 at $0 \mathrm{kPa}$. Also, at the $-100 \mathrm{kPa}$, it was $2-16 \mathrm{CFU} / 20 \mathrm{~mL}$ (average: $12 \mathrm{CFU} / 20 \mathrm{~mL}$ ) with specimen 1 , and $11-55 \mathrm{CFU} / 20 \mathrm{~mL}$ (average: $41 \mathrm{CFU} / 20 \mathrm{~mL}$ ) with specimen 2 . At the condition under $-100 \mathrm{kPa}$, it was $2-9 \mathrm{CFU} / 20 \mathrm{~mL}$ (average: $6 \mathrm{CFU} / 20 \mathrm{~mL}$ ) with specimen 1 , and 8 - 35CFU/20 $\mathrm{mL}$ (average: $27 \mathrm{CFU} / 20 \mathrm{~mL}$ ) with specimen 2. Except a case of $-100 \mathrm{kPa}$ of specimen 1, the decrease of the bacterial count was found in all conditions.

Conclusion: As for the bacteria detection in RO water and dialysate, a considerable case of little quantity of bacteria is expected, and, as for the capture, the membrane filtration method which we can confirm will be a useful method. However, it is easy to cause contamination when we move a process and put a culture media in membrane filtration cartridge in an open space. Also, the filtration by the sudden depression and pressurization might do an underevaluation when bacteria in itself may be wasted on the filtration membrane. Furthermore, the bacterial count measurement needs mastery of techniques, including detailed attention since results are gauged.

\section{9}

\section{Water Chemistry Control of Dialysate Good Purification in Central Dialysis Delivery System (CDDS)}

Fujio Nakamura1, Ichiro Nakagawa², Yuichi Izumi3, Hiroyuki
Goto 4

'Department of Medical Risk, and Crisis Managemant Faculty of Risk and Crisis Management, Chiba Institute of Science, ${ }^{2}$ Ariake Clinic, Shinrakuen Hospital, Japan,

${ }^{3}$ Kobari Hospital, Japan, ${ }^{4}$ Santo Dai-ni Clinic, Japan

Background: The dialysis therapy in the West is personal dialysis therapy. However, dialysis of Japan is a cure by CDDS. Therefore, the system which processes water with a more precise measure against good purification of dialysate is needed. I investigate the measure against dialysate good purification of facilities by CDDS.

Methods: I investigate the water treatment plant of 33 facilities which is performing dialysis therapy. I carried out the Endotoxin activity (ET) of dialysate, and a bacteriological examination. I performed the sampling part by three points, RO water, dialysate (dialysate feed unit), and a dialysis monitoring instrument.

Results: In the membrane filter method, there was no bacterial detection $(0.01 \mathrm{cfu} /$ less than $\mathrm{ml})$, and facilities which had attained simultaneously the Endotoxin activity (ET) below measurement sensitivity (less than $1 \mathrm{EU} / \mathrm{L}$ ) was 11 in 33 facilities. The bacteriological examination performed cultivation for seven days in R2A and a TGEA culture medium. Beta-D glucan (20 pg/less than $\mathrm{ml})$ and a peptide glycan $(0.6 \mathrm{ng} / \mathrm{less}$ than $\mathrm{ml})$ were netative. As for all the facilities(es), RO equipment, Endotoxin retentive filter (ETRF), and an ultraviolet germicidal lamp were installed. There were bacteria and 11 facilities(es) which ET became below in the detection limit. And facilities which is installing UF module before RO equipment or in the back was 8 . While 22 facilities had equipped with ETRF, in 11 facilities, bacteria were detected and, as for ET, below a detection limit did not become and 7 facilities was using ET measure coupler. Wearing of ETRF is indispensable as a measure against good purification of dialisate by CDDS.

Conclusion: In CDDS, establishment of installation of ETRF, the disinfection method, and water treatment equipment is important for good purification of dialysate facilities. 


\section{0 \\ Effect of Elevating Calcium Concentration of Dialysate to Treat Intra-Dialytic Hypotension: A Cross-over Self-Control Trial}

\author{
Zi Li, Lichuan Yang, Xiaolei Cheng, Ping Fu \\ Department of Medicine-Nephrology, West China Hospital \\ of Sichuan University, China
}

Background: Symptomatic intra-dialysis hypotension (IDH) continues to be a common problem. With the use of low calcium (Ca) concentration dialysate in our dialysis center for 5 years, several patients developed IDH occasionally or frequently. Even after multiple therapeutic strategies were induced, IDH still happened in some patients. In this study, we investigated the changes in haemodynamic parameters induced by calcium concentration of dialysis fluid in IDH patients.

Methods: Single-blinded, crossover design was used. Nineteen maintainance hemodialysis patients with IDH were randomized into two groups, beginning by changing the calcium concentration in dialysate either to $1.25 \mathrm{mmol} / \mathrm{L}$ or $1.75 \mathrm{mmol} / \mathrm{L}$. The first 2 -week intervention phase was followed by two-week of wash out with calcium concentration in dialysate as $1.5 \mathrm{mmol} / \mathrm{L}$, switching round the calcium concentration compared to the initial randomization, by a second 2-week intervention phase.

Results: Eighteen patients finished the trial except one patient withdrew due to hypercalcemia. Compared to low calcium concentration $(1.25 \mathrm{mmol} / \mathrm{L})$, medium and high calcium concentration $(1.5 \mathrm{mmol} / \mathrm{L}$ and $1.75 \mathrm{mmol} / \mathrm{L}$ respectively) elevated intradialytic systolic blood pressure $(\mathrm{SBP})$ and heart rate $(\mathrm{P}<0.05)$, decrease the maximum decrease of SBP (the difference between predialytic SBP and the lowest intradialytic SBP), decrease the events of symptomatic IDH. Compared to medium concentration, high calcium concentration dialysate only had lower incident of intradialytic intravenous fluid infusion. No severe disorder of electrolyte were found.

Conclusion: Medium calcium concentration dialysate can effectively improve the hemodynamic stability in IDH patients. Clinical trial with larger sample and longer follew-up is needed.

\section{Dialysis Technologies}

11

\section{Treatment of Dialysis Access Puncture Site Bleeding with the Syvek Patch}

Arshia Ghaffari, Wanwarat Ananthapanyasut, Abid Rizvi, Suma Raju, Eissa Amani, Vito Campese

University of Southern California, USA

Background: Anemia is a common complication of end-stage renal disease (ESRD). Patients with ESRD are at increased risk of bleeding due to multiple factors. These factors can cause difficulty in achieving post-dialysis hemostasis at the puncture sites and con- tribute to post-dialysis bleeding and anemia. Moreover, bleeding delays patient discharge which hinders the flow of patient care. The duration of bleeding and techniques used to stop post-hemodialysis bleeding have received minimal attention. Use of the Syvek patch, a hemostatic product composed of the glycoaminoglycan poly-Nacetyl glucosamine (pGlcNAc), has demonstrated rapid hemostatis in randomized studies of percutaneous procedures in interventional radiology and cardiology. In this study, the patch's effectiveness in achieving hemostasis was assessed for post-dialysis access bleeding.

Methods: This is an open-label pilot study to assess the efficacy of the Syvek patch in accelerating hemostasis (decrease duration of bleeding) at the venous and arterial puncture sites after hemodialysis. Each patient served as his/her own control. Paired t-test was used to compare the mean time to hemostasis in treated versus controls. All statistical analyses are based on two-sided tests $(\& \# 61537 ;=0.05)$. Thirty consecutive ESRD patients who were receiving hemodialysis through an AV fistula or graft at a large outpatient dialysis unit were asked to participate in this study. The Syvek patch was applied to the venous and arterial puncture sites in addition to providing manual pressure. This was compared with providing manual pressure alone. Time to hemostasis for each patient was reported in minutes. The puncture site was checked at odd minute intervals $(3,5,7 \ldots)$ to assess for ongoing bleeding.

Results: As compared to controls, the mean (standard deviation) time to hemostasis at the arterial puncture site decreased from of 9.8 (6.4) to $6.3(4.2) \mathrm{min}$ [ $\mathrm{p}=0.01$ ], a decrease of $34.7 \%$. The mean time to hemostasis at the venous puncture site decreased from 9.1 (6.4 $\min$ ) to $5.6 \mathrm{~min}(3.3 \mathrm{~min})[\mathrm{P}=0.01]$, a decrease of $38.5 \%$. The time to hemostasis for patients on chronic anticoagulation at the arterial and venous puncture sites decreased from 10.0 (2) to 6.2 (1.78) $[\mathrm{p}=0.01]$ and $7.6(2.7)$ to $4.2(1.1) \mathrm{min}[\mathrm{p}=0.03]$ respectively. While the time to hemostasis for patients on antiplatelet therapy did not reach statistical significance, it decreased from 11.3 (7.5) to $7.9 \min (5.7)$ [p $=$ 0.18 ] and $9.9(7.1)$ to $6.7(4.1) \mathrm{min}(\mathrm{p}=0.16)$ at the arterial puncture and venous puncture sites respectively. There were no complications associated with the use of the Syvek patch.

Conclusion: This study demonstrates that use of the Syvek patch in addition to manual pressure at the dialysis puncture sites significantly reduced the time to hemostasis after hemodialysis. This pilot study provides basis for further study of this patch in reducing post-dialysis bleeding, especially in higher risk populations. 
Monitoring of Dialysis

\section{2 \\ Study on Dialysis Condition Input System in Remote Monitoring}

\author{
Suzuki Takashi", Chizuwa Kunihiro², Sugahara Masazumi², \\ Niimura Masaaki², Kawasaki Tadayuki ${ }^{3}$ \\ ${ }^{1}$ Interdisciplinary Graduate School of Science and \\ Technology (Doctoral Program), Shinshu University, \\ ${ }^{2}$ Graduate School of Science and Technology, Shinshu \\ University, ${ }^{3}$ Department of Clinical Engineering, Maeda \\ Institute of Renal Research, Japan
}

Background: The authors are developing an intelligent VPN and working for securing safety and quality of treatment with remote monitoring dialysis information as an application for dialysis medical treatment at concerned facilities and home. If dialysis conditions such as body weight can be transmitted to a dialyzer before dialysis, setting errors would be reduced and safety would increase. This time, we produced experimentally an application to manage dialysis conditions and discussed on usability of the system.

Methods: The system was realized as a Web application. Apache, MySQL and php were used for web servers and a web browser was used for a client. http and socket were used as communication methods. For operation, the web servers were connected to the web browser and followings were performed. (1) Input / save / management of dialysis conditions (2) Body weight before dialysis is transmitted to STEP and quantity of water removal calculated in STEP is transmitted to a dialyzer. (3) Body weight after dialysis is transmitted to STEP. (4) Input / save / management of equipment used for dialysis.

Results: Input and management of dialysis conditions were successfully performed with the proposed application. Moreover, dialysis conditions such as the body weight were successfully transmitted. This is because the system configuration was simple. In other words, the client is available only in a web browser so it was intuitive and easy interface as a major factor. With the body weight before dialysis, the quantity of water removal is calculated in the dialysis support system.

Conclusion: The proposed system enables to perform dialysis smoothly and contributes to security of safety and quality so it is a useful system.

\section{3 \\ Monitoring of Dialysis Dose in Acute Kidney Injury with the Use of Kt Determinated by Ionic Dialisance}

\author{
Rosa Diez Guillermo', Greloni Gustavo', Varela Federico', \\ Crucelegui Soledad", Giannasi Sergio², San Roman \\ Eduardo², Algranati Salomon ${ }^{2}$ \\ ${ }^{1}$ Department of Nephrology-Hospital Italiano de Buenos \\ Aires, ${ }^{2}$ Department of Critical Care-Hospital Italiano de \\ Buenos Aires, Argentina
}

Background: Optimal dose of renal replacement therapy has not yet been established for patients with acute renal injury (AKI) admitted to the intensive care unit (ICU). In daily clinical practice, the final dose is not controlled and we generally do not reach the prescribed dose, unlike what happens with patients with end-stage renal disease. Kt assessed through ionic dialisance provides a direct quantification of dialysis dose without the need for blood sample drawing or dialysate collection. It has been validated for patients with chronic renal failure (CRF) and more recently, for AKI individuals. Given that the difference between the prescribed and the obtained dialysis dose is frequently assessed in CRF, the aim of this study was to measure this parameter using the Kt through ionic dialysance in critical AKI individuals.

Methods: Adult patients with RRT-requiring oliguric AKI admitted to the ICU, who were treated with intermittent and/or extended haemodialysis were admitted into this prospective study. Baseline characteristics including the severity of the AKI according to the "Individual severity index" were recorded. For the purpose of the study, dialysis type and duration were not changed and were delivered according to the prescription of the responsible physician. Fresenius $4008 \mathrm{~S}$ monitors equipped with OCM biosensors (On-line clearance monitoring, Fresenius Medical Care AG) were used. This device is equiped with two conductivity probes to measure, non-invasivaly, effective ionic dialysance. Ultrapure water for haemodialysis was provided by a portable reverse osmosis system. Dialysate fluxes for intermittent and extended hemodialysis were 500 and $300 \mathrm{ml} / \mathrm{min}$, respectively. Sterile powdered bicarbonate was used as buffer. $1.4 \mathrm{~m}^{2}$ helixone membranes were used in all RRTs. Non-tunneled femoral or internal jugular vein, 19 or $16 \mathrm{~cm}$ in length respectively, $11.5 \mathrm{Fr}$ catheters, were placed through Seldinger techniqueUnless contraindicated, heparin was used as anticoagulant at an intravenous loading dose of $1000 \mathrm{U}$ followed by a maintenance hourly dose of $500 \mathrm{U}$. Kt was automatically measured while the prescribed Kt was also calculated. The mean difference between the prescribed and the already delivered dose for all the treatments and for both, intermitent and continuous therapies, were calculated. The differences were compared.

Results: Eighteen patients ( $69 \pm 16$ years old), 14 men, receiving 35 treatments were included. The most common cause of AKI was septic shock, followed by AKI associated with cardiovascular surgery. Patient severity according to the ISI score was $0.67 \pm 0.38$; 13 individuals required mechanical ventilation and 12 were receiving inotropic agents at the time of consultation. Twenty four treatments consisted in intermitent hemodialysis whereas 11 were extended hemodialysis sessions. The mean Kt OCM assessed dialysis dose was $34.9 \pm 10.69 \mathrm{~L}$ and only $69 \%$ of the treatments reached the prescribed KtThe averaged difference was $7.52 \pm 3.24 \mathrm{~L}$, significantly lower for 
extended compared with intermitent dialysis. (5.36 $\pm 1.98 \mathrm{~L}$ vs 9.68 $\pm 1.76 \mathrm{~L} ; \mathrm{p}<0.05)$.

Conclusion: When is adequately monitored, the prescribed dialysis dose is delivered in only $69 \%$ of treatments. Extended hemodialysis reduces the chance of an insuficient dialysis dose. The assessment of dialysis dose through $\mathrm{Kt}$ ionic dialyzance is a useful tool which allows to satisfactorily monitor dialysis treatment.

\section{4 \\ Supplementary Oxygen Promote Hemodilution Leading to Prevent Intradialytic Hypotension}

\author{
Yoshihiro Tange1, Yutaka Isakozawa', Shingo Takesawa', \\ Heihachi Migita1, Shigenori Yoshitake1, Yoshiaki Nishite?2, \\ Yoshio Nagake ${ }^{3}$ \\ ${ }^{1}$ Kyusyu University of Health and Welfare, Nabeoka, ${ }^{2}$ Kinki \\ University, Osaka, ${ }^{3}$ Seiyoukai Nagake Clinic, Osaka, Japan
}

Background: Symptomatic intradialytic hypotension (IDH) affects twenty to fifty $\%$ of end-stage renal disease patients during their regular dialysis therapies. A variety of therapeutic maneuvers have been suggested for the prevention and treatment of IDH. We often found the patients developing IDH kept the cardiovascular stability after administrating supplementary oxygen. When hematocrit (Ht) and oxygen saturation were examined using a monitor for blood volume (BV) after IDH, supplementary oxygen caused the reduction in $\mathrm{Ht}$ and the increased in oxygen saturation leading to the cardiovascular stability. However, the mechanism has not been fully understood.We conducted preliminary study using volunteers to examine the effect of supplementary oxygen on the blood profile and cardiovascular response.

Methods: Thirty healthy volunteers were enrolled in this study after informed consents were obtained. Volunteers positioned supine at rest before the study. A pulse oximeter (N-595 NELLCOR, USA) were continuously monitored to examine the heart rate (HR) and oxyhemoglobine saturation $\left(\mathrm{SpO}_{2}\right)$. After volunteers rested for ten minutes supplementary oxygen was administered through nasal canula 3 liter/min for ten minutes. Blood samples were collected from a vein at the following three points. 1 . before the beginning of supplementary oxygen administration as a baseline 2. 10 mins after the beginning of supplementary oxygen administration. 3.10 mins after the stop of supplementary oxygen. We determined red blood cell count (RBC), white blood cell count (WBC), platelet count (PLT), hematcrit (Ht), hemoglobin $(\mathrm{Hb})$ and calculated mean corpuscular volume (MCV). The venous blood gas were analyzed simultaneously.

Results: HR significantly decreased after supplementary oxygen administration compared to the baseline and returned to the baseline level after stop of oxygen administration. SpO2 significantly increased and decreased before and after supplementary oxygen administration, respectively. RBC, WBC, PLT, $\mathrm{Ht}$ and $\mathrm{Hb}$ significantly decreased after supplementary oxygen administration and 10 mins after stop of supplementary oxygen compared to the baseline levels. MCV decreased significantly after supplementary oxygen administration and 10 mins after stop of supplementary oxygen compared to the baseline levels. From the view point of correlation between $\mathrm{RBC}$ and MCV, high levels of RBC caused the decrease in MCV significantly.
Conclusion: When we previously examined Ht and oxyhemoglobin saturation (Sat) using a monitor for bood volume (CLIT LINE MONITOR, Tokyo, Japan) during hemodialysis treatment, Ht decreased and Sat increased after supplementary oxygen adminidtration. These findings suggested that oxygen administration resulted in hemodilution. The finding of the significant decrease in HR thorough oxygen administration during hemodialysis treatment may show the possibility of making the pulse settle down leading to the cardiac stability. The results in volunteers suggested that the significant reductions in $\mathrm{RBC}, \mathrm{WBC}, \mathrm{Ht}, \mathrm{Hb}$ and PLT after supplementary oxygen administration promoted hemodilution and lead the cardiovascular stability. We concluded that the significant decrease in MCV promoted the hemodilution in this volunteers study suggesting that supplementary oxygen prevented IDH.

\section{5 \\ Measurement of Blood Pressure in the Arteriovenous Shunt for Monitoring Patients Systemic Blood Pressure}

\author{
Sebastian Koball, Michael Hinz, Jan Stange, Steffen \\ Mitzner
}

University of Rostock, Department of Internal Medicine, Nephrology, Rostock, Germany

Background: Hypotension and circulatory failure are common problems during dialysis sessions in patients with high ultrafiltration rates. These problems occur often suddenly without any premonition. This is critical for patients survival and comfort during the dialysis session. Short term noninvasive blood pressure measurement (by the method of Riva-Rocci) is the gold standard for chronic dialysis patients outside the intensive care unit. A short term measurement is often not tolerated by the patient so that first signs of decreasing blood pressure are not detectable. A measurement system which can be performed often in a short term and is not noticed by the patient would be helpful.

Methods: We established a method for the measurement of the blood pressure in the arteriovenous shunt of chronic dialysis patients, while the blood flow in the vascular access has been stopped and the extracorporeal blood flow in the dialysis machine has been redirected on a bypass system. A computer aided control, measurement and analysis system evaluates the absolutely measured pressure values, the slope of the increasing pressure after stopping the blood flow in the vascular access and the the waveform of every single pulse in the arteriovenous shunt. We investigated a cohort of 20 stable dialysis patient with a arteriovenous shunt. The minimum age of the shunt of every patient was 6 months. The systemic blood pressure was measured with an automated device (Dinamap procare 100, GE Helthcare) at upper arm contralateral of the arteriovenous shunt. Both methods were performed every 15 minutes.

Results: The blood pressure in the arteriovenous shunt is correlated with the systemic blood pressure measurement by the method of riva-rocci. Measured values with both methods differed (with lower pressure in the shunt), but the trend of both during a dialysis session was the same. The blood pressure in the shunt depends on shunt characteristics, such as age of shunt, location of shunt, maximum blood flow in the shunt and the prevalence of central stenosis 
in the shunt vein. The measurement procedure is well tolerated by the patient, because it is not noticed by the patient. A very short term measurement is possible. The sensitivity of the detection of decreasing blood pressure values is improved by the additional evaluation of parameters like the slope of the increasing pressure in the arteriovenous shunt after stopping the blood flow in the vascular access and the waveform and the area under the curve of every single pulse in the arteriovenous shunt. The bypass system seems to avoid clotting problems in the extracorporeal circulation during the time the blood flow in the vascular access is stopped. With the bypass system it is possible to use the measurement system with nearly every dialysis device in such a way that it could be established as a stand alone device or it could be implemented in a dialysis device.

Conclusion: The measurement of blood pressure in the arteriovenous shunt of patients on chronic dialysis is an easy to perform, save and comfort method for detecting circulatory failure during a dialysis session. Episodes of severe hypotension can be avoided by early detection of decreasing blood pressure and reducing the ultrafiltration rates. Thus the new system is saving the wellbeing and life of the patient as well as special costs of the treatment of severe hypotension or circulatory failure.

16

\section{The Efficacy of Monitoring Hemodynamics by Multi-Frequent Bioelectrical Impedance Analysis During Dialysis}

\author{
Hiroyuki Sano1, Kenshi Makio², Masae Ueno², Sizuka \\ Numata², Norihisa Hamamoto², Hidekazu Muro², Hakaru \\ Miyazi², Tomotaka Naramura1, Takao lde², Shigeki \\ Minakata2, Shigeyoshi Morimoto², Hisayoshi Sangen ${ }^{2}$ \\ ${ }^{1}$ Ide Clinic, ${ }^{2}$ Sangen Clinic, Kyoto, Japan
}

Background: The decrease of blood volume as caused by the drop of plasma osmotic pressure and water removal often incurs hypotension during hemodialysis (HD). Plasma osmotic pressure defines the water's mobilization between intra and extracellular compartment. Thus if we could monitor this water mobilization, we would be able to acquire a more detailed understanding of the cause of hypotension during HD. Therefore, we examined the relationship between the cause of hypotension and water mobilization during HD. To do this, we used a multi-frequency bioelectrical impedance analysis (MF-BIA). We also evaluated the influence of acetate in dialysate, which is generally considered to be the cause of hypotension during HD.

Methods: In these studies, we used a MLT50 as MF-BIA(SK Medical Electronics Corp., Ltd, Shiga, Japan) with low electrode impedance which enables us to measure total body water (TBW), intracellular fluid (ICF) and extracellular fluid (ECF). We carried out this study on 16 patients whose dry weight were regarded as adequate. 7 of these patients' blood pressures were stabilized during HD:(Group A). 6 of these patients were hypotension-prone during HD:(Group B). We compared a fluctuation of TBW, ICF and ECF between Group A and Group B (Group A vs Group B). The other 3 patients who suffered disabling hemodynamic instability during HD had their conditions dramatically improved after altering their treatment from acetate containing bicarbonate dialysate (BD: sodium $=140$, bicarbonate 30.0, Acetate $=8 \mathrm{mEq} / \mathrm{L}$ ) use to acetate-free dialysate $(A F D$ : Sodium $=140$, Bicarbonate-35.0, Citrate $=2 \mathrm{mEq} / \mathrm{L})$. We compared a fluctuation of TBW, ICF, and ECF under two types of dialysate use(BD vs AFD). During these studies, we did not change any HD conditions which included dialysate flow rate, blood flow rate, and dialyzer.

Results: The results of the MF-BIA showed some notable differences in the fluctuations of ICF. Throughout the treatment, there was a tendency of ICF to decrease in group A and to increase in group B. In the 3 patients who had improved their hemodynamic instability alleviated during $\mathrm{HD}$ after their treatment was altered from BD use to AHD, there was a tendency of ICF to increase under BD use and to decrease under AHD. Based upon these results, it is likely that the tendency of ICF to increase was common to those who were hypotension-prone during HD. There have been many other studies which also corroborate the influence of acetate to hemodynamic instability. Thus, it is likely that acetate suppresses the smooth water transfer from an intracellular space to an interstitial space, and from an interstitial space to an intravascular space due to this enhanced vasopermeability. Therefore, a more vigorous monitoring of water mobilization between intra and extracellular compartments during HD may not only help prevent from hypotension, but may also lead to identifying the very causes of hypotension itself.

Conclusion: A more widespread monitoring of water mobilization by MF-BIA may be vital to comprehending, in detail the hemodynamics of HD.

\section{7 \\ Use of Standard Kt/V for Comparison of Efficiency Between Continuous Renal Replacement Therapies and Intermittent Hemodialysis in Acute Kidney Injury}

Rolando Claure-Del Granado', Etienne Macedo', Sharon Soroko', Glenn M. Chertow', Jonathan Himmelfarb ${ }^{3}$, Alp Ikizler', Emil P. Paganini' ${ }^{5}$, Ravindra L. Mehta ${ }^{1}$

${ }^{1}$ University of California, San Diego, Calif., ${ }^{2}$ Stanford University School of Medicine, Stanford, Calif., ${ }^{3}$ Kidney Research Institute, University of Washington, Seattle, Wash., ${ }^{4}$ Vanderbilt University Medical Center, Nashville, Tenn., ${ }^{5}$ Cleveland Clinic Foundation, Cleveland, Ohio, USA

Background: Standard Kt/V (stdKt/V) is used to measure dialysis efficiency across different types of therapies of variable frequency in patients on chronic dialysis. Two methods are used to calculate stdKt/V (Gotch and Leypold et al) and differ with respect to the number of variables included. In acute kidney injury (AKI) the assessment of dialysis dose across modalities has been limited as effluent volume is employed for delivered dose in CRRT while single pool ( $\mathrm{spKt} / \mathrm{V}$ ) or equilibrated (eKt/V) are used for intermittent hemodialysis (IHD). We evaluated the utility of the Leypold et al. stdKt/V equation to compare the efficiency of IHD and CRRT in patients with AKI. We hypothesized that continuous therapies would be more effective in providing small solute clearance.

Methods: We analyzed data from 1,538 CRRT $24 \mathrm{hr}$ sessions in 244 critically ill patients with at least $48 \mathrm{hr}$ on CRRT and from 610 IHD sessions in 254 critically ill patients from 5 centers included in 
the PICARD study. A detailed description of PICARD inclusion and exclusion criteria, data elements, and data collection and management strategies has been described elsewhere (Kidney Int. 66:1613-21, 2004). Delivered dose was calculated by using stdKt/V Leypold et al. equation: $\operatorname{stdKt} / \mathrm{V}=168 *(1-\exp [-\mathrm{Kt} / \mathrm{V}]) / \mathrm{t} /[(1-\exp [-\mathrm{Kt} / \mathrm{V}]) /(\mathrm{Kt} / \mathrm{V})$ $+168 /(\mathrm{N} * \mathrm{t})-1]$, and was adjusted for each type of CRRT as shown by Diaz-Buxo JA and Perez J (Artif Organs 30:178-185, 2006). We compared the efficacy of the following treatment regimens: IHD (1 to 7 treatments per week), continuous veno-venous hemofiltration (CVVH), continuous veno-venous hemodialysis (CVVHD) and continuous veno-venous hemodiafiltration (CVVHDF).

Results: A good correlation was found between $s p K t / \mathrm{V}$ (Daugirdas equation) and Leypold et al. stdKt/V equation $\left(r^{2}=0.961\right.$; $\mathrm{p}<0.001$ ). The median (interquartile range $[\mathrm{IQR}]$ ) of delivered single pool Kt/V (spKT/V) for a single IHD treatment was $0.85(0.63-1.15)$. Frequency of intermittent hemodialysis sessions ranged from 1 to 7 treatments per week. Higher frequency of IHD treatments per week was associated with increased delivered stdKt/V (median [IQR]): from $0.58(0.47-0.67)$ for 1 treatment; $1.26(0.92-1.47)$ for 2 sessions; $1.83(1.50-2.15)$ for $3 ; 2.39$ (1.91-2.84) for $4 ; 2.67(2.17-3.19)$ for 5 ; 3.15 (2.60-3.97) for 6 ; and 3.77 (3.1-5.2) for 7 treatments per week respectively. A significant correlation was found between dose expressed as $\mathrm{mL} / \mathrm{kg} / \mathrm{hr}$ and $\mathrm{stdKt} / \mathrm{V}\left(\mathrm{r}^{2}=0.976 ; \mathrm{p}<0.001\right)$. Among CRRT modalities the median (IQR) of delivered $\mathrm{ml} / \mathrm{kg} / \mathrm{hr}$ and stdKt $/ \mathrm{V}$ were: CVVH $21.1 \mathrm{~mL} / \mathrm{kg} / \mathrm{hr}(12.3-26.1)$ and stdKt/V 6.5 (3.9-8.1); CVVHD $19.3 \mathrm{~mL} / \mathrm{kg} / \mathrm{hr}(15.1-24.2)$ and stdKt $/ \mathrm{V} 5.8$ (4.6-7.4); and CVVHDF $27.5 \mathrm{~mL} / \mathrm{kg} / \mathrm{hr}(20.1-35.8)$ and stdKt $/ \mathrm{V} 9.1$ (7.1-11.5) respectively. Continuous veno-venous hemodiafiltration offered the highest efficiency among continuous renal replacement therapies ( $p$ $<0.001)$. Continuous therapies provide higher delivered dose with a median (IQR) stdKt/V of $7.6(5.6-9.9)$ as compared to intermittent modalities stdKt/V of $1.9(1.5-2.4)$ modeled for a 3 times per week treatment $(\mathrm{p}<0.001)$. This difference in providing a more effective clearance of small solutes remains significant even after modeling the IHD modality for a dialysis intensity of 7 treatments per week; stdKt/V of 4.6 (3.6-5.8) for IHD as compared to stdKt/V of 7.6 (5.69.9) for CRRT [p $<0.001]$.

Conclusion: Standard Kt/V allows comparison of delivered dose across different modalities of renal replacement therapies and treatment schedules as it is normalized for the duration and frequency of therapy. Continuous therapies provide the best opportunity for providing a sustained dialysis dose over time in patients with AKI. Additional studies are required to evaluate the utility of $s t d K t / v$ for molecules other than urea nitrogen, and correlate delivered dose with outcomes from AKI.

\section{Hemodialysis}

\section{8 \\ Clinical Importance of Endotoxemia in Maintenance Hemodialysis Patients}

\author{
Jae-Yuen Park, Sung-Eun Hu, Kyu-Beck Lee, Hyang Kim \\ Division of Nephrology, Kangbuk Samsung Hospital, \\ Sungkyunkwan University, School of Medicine, Seoul, \\ Korea
}

Background: Inflammation, malnutrition and artherosclerosis are common symtoms in end stage renal disease (ESRD) and they are related to poor prognosis. Recently endotoxemia is reported as a risk factor of inflammation and atherosclerosis. The aim of this study is to collect the level of endotoxin from ESRD patients on hemodialysis and to find the association between endotoxin level and inflammation, nutrition, and the indicators of atherosclerosis.

Methods: From 64 patients with ESRD on maintenance hemodialysis, we collected patients characteristics and laboratory measurements by medical records and measured the level of endotoxin with LAL method (Limulus amebocyte lysate). We analyzed the association between the level of endotoxin and the marker of nutrition (albumin, malnutrition-inflammation score(MIS)), inflammation (hs-CRP), atherosclerosis (aortic arch calcification (AAC), and ba$\mathrm{PWV}$, overhydration). And we divided the samples into the positive group (the level of endotoxin $>0.05 \mathrm{EU} / \mathrm{ml}$ ) and negative group (< $0.05 \mathrm{EU} / \mathrm{ml}$ ) and compared the marker of nutrition, inflammation, and atherosclerosis.

Results: 1. The level of endotoxin in maintenance hemodialysis was $0.09 \pm 0.14 \mathrm{EU} / \mathrm{ml}$, and the positive group ( the level of endotoxin $>0.05 \mathrm{EU} / \mathrm{ml}$ ) was eleven $(17 \%)$. 2. There were no correlations between the level of endotoxin and the level of albumin, MIS, hsCRP, AAC, ba-PWV, overhydration ( $p>0.05)$, but there was correlation between the level of endotoxin and the duration of maintenance hemodialysis $(\mathrm{r}=0.34, \mathrm{p}=0.03) .3$. Between the negative $(\mathrm{N}=54)$ and the positive group $(\mathrm{N}=11)$, there were no associations in age $(58 \pm 12$ vs $58 \pm 13$ year), Charlson comorbidity index $(5.2 \pm 2.0$ vs $5.5 \pm 2.2)$, albumin $(3.9 \pm 0.3$ vs $4.0 \pm 0.3 \mathrm{~g} / \mathrm{dL})$, MIS $(6.5 \pm 3.1$ vs $5.9 \pm 3.1)$, hsCRP $(0.37 \pm 0.80$ vs $0.56 \pm 0.74 \mathrm{mg} / \mathrm{dL}), \mathrm{AAC}(7.8 \pm 4.4$ vs $9.3 \pm 2.7)$, baPWV $(1942 \pm 566$ vs $2189 \pm 499 \mathrm{~cm} / \mathrm{sec})$, and overhydration $(1.7 \pm 1.8$ vs $1.2 \pm 3.1 \mathrm{~L})(\mathrm{p}>0.05)$. However, in the positive group, the duration of hemodialysis was significantly longer ( $37 \pm 36$ vs $64 \pm 49$ month) $(\mathrm{p}=0.05)$.

Conclusion: The increased endotoxin level was observed in some maintenance hemodialysis patients. The increased endotoxin level was related to the duration of maintenance hemodialysis, but not to the nutrition, inflammation, and atherosclerosis. Further prospective study is needed to clarify the role of endotoxin in the development of nutrition, inflammation and atherosclerosis in ESRD patients. 
19

\section{Accuracy of Body Composition Monitor to Detect Changes of Body Fluid Status According to Ultrafiltration Volume During a Hemodialysis Treatment}

Jae-Yuen Park, Sung-Eun Hu, Kyu-Beck Lee, Hyang Kim

Division of Nephrology, Kangbuk Samsung Hospital, Sungkyunkwan University, School of Medicine, Seoul, Korea

Background: Accurate determination of fluid status in hemodialysis (HD) patients is essential to blood pressure and cardiovascular complications. Bioimpedance spectroscopy (BIS) is a technique that measures the impedance of body tissues over wide frequency ranges and a promising method to assess to extracellular water (ECW) and total body water (TBW). The aims of this study were to assess (1) the accuracy of new BIS device (BCM- Body Composition Monitor, Fresenius Medical Care) to detect the changes in the body fluid status during a HD treatment, (2) the influence of several factors (age, sex, diabetes, vascular access, overhydration $(\mathrm{OH})$ ) on the accurate of BCM.

Methods: 39 maintenance HD patients were measured with $\mathrm{BCM}$ before a HD, at $60 \mathrm{~min}, 120 \mathrm{~min}, 180 \mathrm{~min}$ during a $\mathrm{HD}$ and at the end of a HD, 30 min after a HD over the total 6 times. The ultrafiltration volumes (UFV) were compared to the changes of TBW, $\mathrm{ECW}, \mathrm{OH}$ over HD treatment times for each patient. We evaluated the accuracy of BCM according to several factors.

Results: There were good correlations between UFV and delta $\mathrm{OH}($ postOH-preOH), delta TBW(postTBW-preTBW), delta ECW(postECW-preECW) measured after a HD, and good correlations between UFV and delat TBW, delta ECW during a HD. The correlation coefficients between UFV and delta $\mathrm{OH}$, delta TBW, delta ECW were $0.42,0.45,0.41(\mathrm{p}<0.05)$ (postHD $0 \mathrm{~min}), 0.56,0.47,0.52$ $(\mathrm{p}<0.05)$ (postHD $30 \mathrm{~min}$ ). Using Bland-Altman plot, the $95 \% \mathrm{CI}$ of agreement between UFV and delta $\mathrm{OH}$ ranged from $-2.47 \mathrm{~L} \sim 1.51 \mathrm{~L}$ (postHD $0 \mathrm{~min}$ ), $-2.40 \mathrm{~L} \sim 1.28 \mathrm{~L}$ (postHD $30 \mathrm{~min}$ ). The correlations between UFV and delta OH, delta TBW, delta ECW after a HD were better in patients with diabetes, $\mathrm{preOH}<2 \mathrm{~L}$ and no difference in age, sex and vascular access.

Conclusion: Our data suggest the $\mathrm{OH}, \mathrm{TBW}$ and ECW measured by BCM, accurately estimate the changes in the fluid status after a HD. BCM was more accurate in patient with diabetes, preOH $<2 \mathrm{~L}$.

\section{0}

The Harmful Effect of Cigarette Smoking on Atherosclerosis in Japanese CKD Stage 5 Patients

\author{
Sawako Kato', Shoichi Maruyama', Yoshinari Yasuda', \\ Yoshinari Tsuruta ${ }^{2}$, Akira Ito ${ }^{3}$, Fumi Kato ${ }^{3}$, Nami Takai ${ }^{3}$, Yukio \\ Yuzawa ${ }^{4}$ \\ ${ }^{1}$ Nagoya Univ. Graduate School of Medicine, ${ }^{2}$ Meiyo \\ Clinic, ${ }^{3}$ Masuko Memorial Hospital, ${ }^{4}$ Fujita Health Univ. \\ School of Medicine, Japan
}

Background: Cigarette smoking (CS) is an established risk factor of atherosclerosis which leads to cardiovascular disease (CVD) due to endothelial dysfunction in general population. CVD is a major mortality and morbidity in chronic kidney disease (CKD) patients and CS has been also identified as a progression factor of (CKD). However, it has not yet been clarified how harmful effect of CS plays a role on atherosclerosis in Japanese CKD stage 5 patients.

Methods: We have investigated 67 Japanese CKD stage 5 patients (42 males, mean age $59.5 \pm 11.1$ years) who have just started renal replacement therapy (RRT) from June 2007 to March 2010 at Masuko memorial Hospital and Meiyo Clinic in Aichi prefecture, Japan. This study is a sub-analysis of our ongoing prospective study approved by the Ethics Committee of Nagoya University Graduate School of Medicine and informed consent was obtained from all patients . The clinical laboratory parameters related to risk factors of CVD were obtained. To assess vascular stiffness as an index of atherosclerosis, brachial ankle pulse wave velocity (baPWV) was measured with a pulse pressure analyzer; Form PWV/ABI BP-203RPE (Nihon Colin Co., Ltd. Tokyo, Japan). All statistical analyses were performed using statistical software JMP version 8 for Macintosh (SAS).

Results: The 38 (34.3\%) out of 67 patients had CS history (14 current smoker/ 24 ex-smoker). Compare with nonsmokers (Group non-CS), the patients with history of CS (Group CS) had significant higher CRP levels $(0.63(0.016-5.54)$ vs. $0.26(0.001-2.77) \mathrm{mg} /$ $\mathrm{dL} ; \mathrm{p}=0.0085)$ and higher ferritin levels $(143.7(18.0-879.0)$ vs. $101.4(13.0-812.0) \mathrm{ng} / \mathrm{mL} ; \mathrm{p}=0.013)$ although they had also lower total cholesterol levels (156.8(90-248) vs. 183.6(99-269) mg/ $\mathrm{dL} ; \mathrm{p}=0.007)$ and lower HDL cholesterol levels (40.7(22.5-79) vs. $51.3(23.8-92) \mathrm{mg} / \mathrm{dL} ; \mathrm{p}=0.0035)$. Whereas no difference in age, prevalence of diabetes, ACEi/ARB use and statin use between two groups, group CS had significant higher baPWV levels $(19.09 \pm 0.60$ vs. $16.81 \pm 0.68 \mathrm{~m} / \mathrm{sec} ; \mathrm{P}=0.013)$, which suggests more severe stiffness of vessels. Multiple regression analysis for baPWV gave a significant model with CS, age, BMI, mean BP, gender, DM as the dependent variables $(\mathrm{R} 2=0.35, \mathrm{p}=0.0005)$. In this model, $\mathrm{CS}$, age and mean $\mathrm{BP}$ showed confirmed contribution to baPWV levels (CS; 285.4 \pm 100.6 , $\mathrm{P}=0.0064$, age; $12.6 \pm 3.8, \mathrm{P}=0.0017$, mean $\mathrm{BP} 7.0 \pm 3.2, \mathrm{P}=0.0034$, coefficient $\pm \mathrm{SE}$, respectively).

Conclusion: In CKD patients, chronic inflammation is well known to be deeply associated to atherosclerosis which results in high incidence of CVD and premature mortality. In this study, the patients with history of CS showed increased CRP levels and higher baPWV levels. We suggest that CS may be one of accelerators of atherosclerosis through induction of inflammation in CKD patients. 


\section{1 \\ Assessment of Vancomycin and Gentamicin Clearance by Dialysis Using in vitro and in vivo Systems}

\author{
Nathan Pinner ${ }^{1}$, Robert Canada², Joyce Broyles ${ }^{3}$, Joanna \\ Hudson ${ }^{4}$ \\ ${ }^{1}$ Auburn University Harrison School of Pharmacy \& \\ University of Ala., School of Medicine, Tuscaloosa, \\ Ala., ${ }^{2}$ University of Tenn., Dept. of Medicine, Memphis, \\ Tenn., ${ }^{3}$ Methodist University Hospital, Memphis, Tenn., \\ ${ }^{4}$ University of Tenn., Depts of Clinical Pharmacy \& \\ Medicine, Memphis, Tenn., USA
}

Background: Advances in hemodialysis (HD) have increased potential for drug removal, but quantifying drug clearance for all drug-dialyzer conditions is impractical. The purpose of this study was to: 1) Determine dialysis clearance (CLD) of vancomycin (V) and gentamicin $(\mathrm{G})$ by dialyzers used for intermittent hemodialysis (HD) and sustained low efficiency dialysis (SLED) using in vivo and in vitro systems, and 2) evaluate application of the in vitro system to assess CLD of other agents.

Methods: In vivo system - End-stage renal disease patients receiving $\mathrm{V}$ and/or $\mathrm{G}$ were enrolled. HD was performed using the Optiflux F180NR dialyzer. Simultaneous arterial (Ca) and venous $(\mathrm{Cv})$ samples were collected at regular intervals and the mean CLD was calculated as $((\mathrm{Ca}-\mathrm{Cv}) / \mathrm{Ca}) \times \mathrm{BFR} \times(1$-Hematocrit) where BFR is blood flow rate. In vitro system- The dialysis system consisted of a phosphate buffer solution with V $(100 \mathrm{mg} / \mathrm{L}), \mathrm{G}(60 \mathrm{mg} / \mathrm{L})$ and albumin $(2 \mathrm{~g} / \mathrm{L})$ added to mimic in vivo conditions. Urea (U) and creatinine $(\mathrm{Cr})$ were added as marker compounds. Two Fresenius polysulfone dialyzers were studied: the F180NR used for intermittent HD and the F50 used for sustained low efficiency dialysis (SLED). Simulated HD conditions: dialysate flow rate (DFR) $500 \mathrm{~mL} / \mathrm{min}$, "blood" flow rate (BFR) $200 \mathrm{~mL} / \mathrm{min}$. Simulated SLED conditions: DFR $350 \mathrm{~mL} / \mathrm{min}$, BFR $150 \mathrm{~mL} / \mathrm{min}$. In vitro dialysis was performed for 90 minutes in triplicate for each condition. Simultaneous arterial and venous samples were collected at regular intervals to determine mean CLD as CLD = BFR $(\mathrm{Ca}-\mathrm{Cv}) / \mathrm{Ca}$.

Results: In vivo results -The in vivo CLD ( $\mathrm{mL} / \mathrm{min}$ ) of $\mathrm{V}$ was $103 \pm 15$ at a plasma flow rate of $219 \pm 34 \mathrm{~mL} / \mathrm{min}$ ( $\mathrm{n}=10$ patients). CLD for $G$ was $132 \pm 25$ at a plasma flow rate of $234 \pm 31 \mathrm{~mL} / \mathrm{min}$ ( $\mathrm{n}=7$ patients). In vitro CLD for the F180 dialyzer at a BFR of 200 was $131 \pm 3 \mathrm{~mL} / \mathrm{min}$ for $\mathrm{V}$ and $154 \pm 3 \mathrm{~mL} / \mathrm{min}$ for $\mathrm{G}$. In vitro CLD for the F50 dialyzer at BFR of 150 was $72 \pm 9 \mathrm{~mL} / \mathrm{min}$ for $\mathrm{V}$ and 84 $\pm 11 \mathrm{~mL} / \mathrm{min}$ for $\mathrm{G}$. CLD for the marker compounds $\mathrm{U}$ and $\mathrm{Cr}$ was consistent with manufacturer's data for the F180 (195 \pm 2 for U and $187 \pm 3$ for $\mathrm{Cr}$ ) and for the F50 (129 \pm 17 for $\mathrm{U}$ and $120 \pm 14$ for $\mathrm{Cr}$ ) at similar flow conditions providing additional data to validate the in vitro system.

Conclusion: This study provides CLD data for V and G from the in vitro system similar to in vivo results supporting the value of in vitro evaluation of drug elimination. The in vitro technique may provide estimates of removal by dialysis for the growing number of drug products to assist in optimization of drug regimens in dialysis patients. Variation in observations is likely due to limitations of in vitro conditions, but estimates are reasonable to assist with drug regimen design.

28th Annual Meeting of the International Society of Blood Purification (ISBP)

\section{2 \\ Mechanisms of Uremia-Induced Down Regulation of ApoA-I Expression}

Hamid Moradi, Hamid Said, Nosratola Vaziri

University of California, Irvine, Calif., USA

Background: Atherosclerotic cardiovascular disease is a major cause of mortality in CKD patients. Decreased HDL level and defective HDL-mediated reverse lipid transport contribute to the atherogenic diathesis in this population. The primary cause of HDL deficiency in CKD is reduction of apoliprotein A-I which is the principal component of HDL. Plasma ApoA-I is reduced in dialysis patients and hepatic ApoA-1 mRNA expression is decreased in the uremic rats. This study explored the mechanism/s by which uremia down-regulates ApoA-I expression.

Methods: HepG2 cells were incubated for 48 hours in media containing whole serum or serum subfractionation (prepared using Amicon filters with different size selectivities) from normal control subjects and ESRD patients before and after hemodialysis. Cells and culture media were then sparated and used for measurement of ApoA-I (ELISA) and ApoA-I mRNA (real-time PCR). ApoA-1 production was calculated from ApoA-1 levels in the fresh and harvested media.. The effect of the test sera on ApoA-I promoter activity was measured using transfection with a luciferase promoter construct containing the -2096 to +293 segment of ApoA-I gene. RNA stability was assessed in presence of actinomycin D.

Results: Exposure to uremic serum resulted in a 5-6 folds reduction in the ApoA-I mRNA expression and a significant reduction of ApoA-I level in the culture media $(137+40$ vs.38+5). This effect was fully reversed when uremic serum was replaced with the control serum. Transfection studies revealed no difference between uremic and normal sera on ApoA-I gene promoter activity. However exposure to uremic serum significantly reduced RNA stability. Fractionation studies revealed that the negative effect of uremic serum on ApoA-I mRNA abundance resides in the fraction containing molecules larger than $30 \mathrm{Kd}$ but not the fraction containing molecules $<30 \mathrm{Kd}$. This supposition was supported by the observation that post-dialysis serum samples exerted an equally potent inhibitory effect on ApoA-I mRNA abundance as that found with the pre-dialysis serum samples.

Conclusion: Uremia causes a significant reduction in ApoA-I mRNA abundance by lowering RNA stability without altering ApoA-I promoter activity. The negative effect of uremic milieu on ApoA-I mRNA abundance/stability resides in the fraction containing molecules larger than $30 \mathrm{Kd}$ which are not removable by hemodialysis. 
23

\section{Effect of Hemodialysis on the Composition of Exhaled Breath}

\author{
Hyun Ji (Julie) Lee', Madeleine V. Pahl'2, Nick D. Vaziri², \\ Donald R. Blake1 \\ ${ }^{1}$ Department of Chemistry, University of California, Irvine, \\ ${ }^{2}$ Medicine, Division of Nephrology and Hypertension, USA
}

Background: Advanced kidney disease results in profound dysregulation of acid-base, mineral, fluid and electrolyte metabolism, accumulation of nitrogenous waste ducts, oxidative stress and inflammation which if untreated culminates in death. Via convective and diffusive transport of fluids and solutes, intermittent hemodialysis (HD) treatment partially restores fluid/electrolytes/mineral and acid-base balance and removes nitrogenous waste products, thereby extends patients' lives. However, the inherent exposure of blood to tubing and dialyzer membranes and influx of impurities from the dialysis fluid as well as mechanical stress caused by roller pump during dialysis procedure lead to activation of leukocytes, platelets and complement cascade. Together these events cause rapid and dramatic changes in the volume and composition of body fluids, cellular metabolism and pulmonary function. While the effect of renal failure and dialysis on body fluids volume and composition is well known, their effect on the chemical contents of breath has received little attention. Several investigators have reported elevated level of biomarkers of oxidative stress such as ethane and n-pentane in patients undergoing HD [Ward et al.(2003), Handelman et al.(2003), Abuja et al.(2001), \& Patterson et al.(2007)]. However, others (Gorham et al. 2009) suggested that breath ethane did not appear to be biomarker of oxidative stress. The present study was designed to: 1- identify gases whose concentration either decreases or increases in the expired breath during the HD; 2 determine the effect of inter-dialytic interval on breath concentrations such gases; and 3-determine if the exhaled ethane in HD patient is a reliable biomarker of lipid peroxidation in the course of HD.

Methods: Ten patients with end-stage renal disease, ESRD, (four men and six women, aged $20 \sim 80$ years) were studied during HD procedure conducted twice at 72 and 48 hour-inter-dialytic intervals. On each occasion, 20 expired breath and 18 room air samples were collected using evacuated 1.9L stainless steel canister. The samples were analyzed on a six column/detector gas chromatography system to qualify about 100 different chemicals including hydrocarbons, halocarbons, oxygenates, and alkyl nitrates.

Results: Breath samples in the ESRD patients contained significant concentrations of methanol which steadily declined during the course of HD procedure. On each occasion methanol concentrations was greater in samples obtained following the long $(72 \mathrm{hr})$ than short (48 hr) inter-dialytic interval. Simultaneous examination of the room air showed insignificant levels of methanol which remained constant. These observations point to endogenous as opposed to exogenous source of the detected methanol in ESRD patients, its accumulation during the inter-dialytic interval and its removal and /or suppressed production by HD therapy. In addition to methanol significant amounts of ethane were detected in the patients' exhaled breath throughout the HD procedure. However, unlike methanol, ethane levels in patients' exhaled breath paralleled those of the dialysis center's room air.

Conclusion: Relationship between exhaled breath methanol concentration and inter-dialytic interval suggests that renal failure results in retention of methanol. The steady reduction of exhaled breath methanol concentration during HD treatment points to its efficient clearance by HD. The parallel change in exhaled breath and room air ethane concentrations during dialysis treatment tends to diminish its validity as a reliable endogenous biomarker in this setting.

24

\section{Risk Factors for Death in a Hemodialysis Population: Experience of a Dialysis Unit Covered by Private Insurance}

\author{
Ana Cristina da Carvalho Matos, Maria Claudia Cruz \\ Andreoli, Adriano Luiz Ammirati, Miguel Angelo Goes, \\ Erika Rangel, IIson lizuka, Fabiana Dias Carneiro, Ana \\ Claudia Mallet Ramos, Rosana Maria Cardoso, Christiane \\ Karan, Bento Fortunato C. dos Santos \\ Dyalisis Unit - Albert Einstein Hospital, São Paulo, Brazil
}

Background: Currently 87000 patients have been on chronic dialysis program in Brazil, $90 \%$ of them are on hemodialysis. There are 600 dialysis units which are accredited by the Brazilian Society of Nephrology and a peculiar aspect of this is $87 \%$ of them have their reimbursement from the Brazilian government and $13 \%$ of them have private insurance coverage that reimburses dialysis treatment. In this study, our aim is to analyze risk factors for death in a hemodialysis population of a hospital in Brazil who have a private insurance and compare our data with those of annual survey of the Brazilian Society of Nephrology 2008.

Methods: We analyzed retrospectively all patients from the Dialysis Unit of Albert Einstein Hospital who had been on chronic hemodialysis program for at least 3 months, during the period from February 2000 to February 2009. Demographic characteristics, medical history, predialysis medical care and laboratory data were collected from medical records. Patients were followed from enrollment until the first of following events: death, transplant, transfer out of the facility, renal function recover or return to conservative treatment. Our results were compared to Brazilian registry data according to Brazilian Society of Nephrology 2008.

Results: A total of 131 patients were enrolled in this study with a mean age of $65.6 \pm 15.3$ years, 40 of patients were female and 53 presented diabetes mellitus as the cause of ESRD. According to ICED distribution, almost $70 \%$ of patients had moderate and severe comorbidities.During nine years of the follow-up period, we observed 46 (35.1\%) deaths being 19 of them (41.3\% of deaths) within first year. Our most frequent immediate cause of death was infection. In a univariate analysis, clinical variables significantly associated with death were: diabetes as cause of ESRD ( $p=0.025)$, age $>70$ yrs $(p<0.0001)$, ICED 3 versus $1(\mathrm{p}=0.001)$, ischemic cardiac disease in admission $(\mathrm{p}<0.0001)$, arrthymia in admission $(\mathrm{p}=0,020)$, diabetes in admission $(p=0,007)$ and congestive heart failure in admission $(p=0,018)$. Hemoglobin at 3 months $>11 \mathrm{~g} / \mathrm{dl}$ had a protective effect $(\mathrm{p}=0,017)$. In a multivariate analysis the best model proposed was that one which considered age $>70$ years $(\mathrm{p}=0.02)$ and the presence of ischemic cardiac disease at admission $(\mathrm{p}=0.03)$ adjusted by hemoglobin values at 3 months $>11 \mathrm{~g} / \mathrm{dl}(\mathrm{p}=0.19)$. In comparison with annual survey of the Brazilian Society of Nephrology of 2008, our sample of patients with private insurance was older $(50.4 \%>70$ years versus $36.3 \%>$ 65 years), presented more diabetes as a comorbidity (48.8\% against 
$25 \%)$ and had more often diabetes as cause of ESRD (40.4\% versus $26 \%$ ). Considering specific cardiovascular comorbidities at admission, our sample had more ischemic cardiovascular disease $(50.4 \%$ versus $8.2 \%$ ), congestive heart failure $(46.6 \%$ versus $10.4 \%)$, cerebral vascular disease $(19.8 \%$ versus $3.7 \%)$ and ischemic peripheral disease $(19.8 \%$ versus $5.2 \%)$. Almost $60 \%$ of our patients had catheter as vascular access at admission versus $66.7 \%$ in Brazilian registry data. Only $50 \%$ of our patients received pre-ESRD nephrology care and this information was not available in Brazilian registry data. Cardiovascular disease was the first cause of death in Brazilian registry data.

Conclusion: In conclusion, age and ischemic cardiac disease were important risk factors for death in our sample and a hemoglobin $>11 \mathrm{~g} / \mathrm{dl}$ at 3 months had a positive impact on survival. Also we found in peculiar sample of hemodialysis patients who have private insurance in Brazil that these patients are more often older, diabetics and with more and severe comorbidities than patients from public dialysis units. This population had an inefficient pre-ESRD nephrology care, with a high rate of late referral and catheter is the most frequent vascular access at the start of hemodialysis treatment, probably contributing for the most part of deaths within the first year of dialysis treatment.

\section{5 \\ Serum Phosphorus Levels Improve After Transition to In-Center Nocturnal Hemodialysis}

Robert I. Lynn', Linda Francisco ${ }^{2}$, Shane Simon ${ }^{2}$, Abbe Volz ${ }^{2}$, Karen Spach ${ }^{2}$, Ronald Levine ${ }^{2}$, Robert Provenzano ${ }^{2}$

${ }^{1}$ Nephrology \& Hypertension Associates, Bronx, NY, ${ }^{2}$ DaVita Inc., Denver, Colo., USA

Background: Maintaining the KDOQI-recommended (3.5 - 5.5 $\mathrm{mg} / \mathrm{dL})$ serum phosphorus $\left(\mathrm{PO}_{4}\right)$ levels is difficult for hemodialysis patients despite dietary counseling and an ever-expanding selection of oral phosphate binders. Hyperphosphatemia can lead to secondary hyperparathyroidism and increased mortality from cardiovascular disease. Given the dialysis time required to re-establish phosphate balance, we hypothesized that in-center nocturnal hemodialysis (NHD), with its longer treatment times, would result in substantially greater $\mathrm{PO}_{4}$ removal, resulting in a lower serum $\mathrm{PO}_{4}$.

Methods: In a retrospective, longitudinal cohort study $(n=418)$, we evaluated parameters of bone and mineral metabolism prior to patient conversion from ICHD to NHD (baseline, mean 1 through 6 months before nocturnal treatment) to these same parameters following the start of NHD (final, mean 4 through 9 months post modality change).

Results: Patients received 3 sessions per week on both ICHD and NHD. Changes in median treatment time and bone and mineral metabolism parameters after conversion to NHD are shown (Table).

Conclusions: After conversion to NHD, patients had lower serum $\mathrm{PO}_{4}$ and $\mathrm{Ca} \times \mathrm{P}$ product compared to their levels on ICHD. We believe the lower levels of $\mathrm{PO}_{4}$ are a result of the longer dialysis sessions with NHD. The decrease in serum $\mathrm{PO} 4, \mathrm{Ca} \times$ Phos product may result in long-term cardiovascular benefits for the NHD patient. In this study we did not control for binder or Sensipar usage. Analysis
Table 1

\begin{tabular}{lccl}
\hline & ICHD (baseline) & NHD & p-value \\
\hline $\begin{array}{l}\text { Median treatment time (hrs/ } \\
\text { session) }\end{array}$ & $4.0 \pm 0.04$ & $7.6 \pm 0.04$ & $<0.001$ \\
Phosphorus $(\mathrm{mg} / \mathrm{dL})$ & $5.8 \pm 0.03$ & $5.1 \pm 0.04$ & $<0.001$ \\
Calcium $(\mathrm{mg} / \mathrm{dL})$ & $9.17 \pm 0.71$ & $9.14 \pm 0.77$ & $\mathrm{NS}$ \\
$\begin{array}{l}\text { Ca } \times \text { P product }\left(\mathrm{mg}^{2} / \mathrm{dL}^{2}\right) \\
\text { Paracalcitol }(\mu \mathrm{g} / \mathrm{patient} /\end{array}$ & $52.9 \pm 0.3$ & $46.7 \pm 0.3$ & $<0.001$ \\
month) & $43.1 \pm 0.9$ & $51.4 \pm 1.0$ & $<0.001$ \\
Parathyroid hormone $(\mathrm{pg} / \mathrm{ml})$ & $472.3 \pm 10$ & $448.9 \pm 10$ & $\mathrm{NS}$ \\
\hline
\end{tabular}

of binder types and amounts, prior to and after the initiation of NHD, is planned in order to confirm that the nocturnal dialysis treatments were responsible for the observed results.

\section{6 \\ An Innovative Pharmacy Management Program Reduces Racial Disparities by Improving Medication Access}

Allen R. Nissenson, Josh Golomb, Joe Weldon, Steve Wilson

DaVita Inc, Denver, Colo., USA

Background: In CKD, racial and ethnic minorities experience disparities in access to and delivery of care. It is possible these disparities also exist in the in-center hemodialysis (ICHD) population given they are prescribed an average of 7-9 medications/day. In an effort facilitate medication access for ICHD patients and thereby potentially enhance medication adherence, DaVita RxSM was developed. This innovative program delivers prescribed medications to patients within the dialysis centers. We hypothesized in-center medication delivery through DaVita Rx would improve medication adherence. We tested this by assessing serum phosphorus levels as an indicator of clinical outcomes for DaVita Rx patients.

Methods: We conducted a retrospective analysis of serum phosphorus levels in 8,120 ICHD patients across 373 dialysis centers. We analyzed phosphorus levels by race and ethnicity in a subgroup of 1094 patients. Serum phosphorus was analyzed 9 months prior to enrollment in DaVita Rx and 1 year after enrollment in the program. We performed a mixed-model analysis on estimated of mean values at each quarterly time point. We used least squares estimates to calculate mean values.

Results: Compared to DaVita at large, Hispanics (+13\%) and African Americans (+9\%) were over-represented in the DaVita Rx pharmacy program population while Caucasians were under-represented $(-20 \%)$. The percent of patients within KDOQI-recommended serum phosphorus guidelines $(3.5-5.5 \mathrm{mg} / \mathrm{dL})$ increased $6 \%$ in patients participating in DaVita Rx compared to $3 \%$ in DaVita at large. This effect was even more pronounced in African Americans (8\% DaVita Rx vs. 2\% DaVita at large).

Conclusion: These results indicate that improved medication management through a program like DaVita Rx can improve biochemical outcomes in minority populations. The disproportionate use of DaVita Rx by African American and Hispanic populations sug- 
gests an unmet need in these groups. Differences in clinical outcomes among various ethnic/racial groups may be overcome with improved care processes.

27

\section{Associations of Flow Mediated Dilation and Pulse Wave Velocity with Intradialytic Hypotension and Hypertension}

\author{
Ruth Dubin ${ }^{1}$, Christopher Owens ${ }^{2}$, Carmen A. Peralta1, \\ Nelson Schiller ${ }^{3}$, John Teerlink ${ }^{3}$, Kirsten Johansen ${ }^{1}$ \\ ${ }^{1}$ University of California San Francisco, Dept. of \\ Medicine, Division Nephrology, ${ }^{2}$ University of California \\ San Francisco, Dept. of Vascular Surgery, ${ }^{3}$ University \\ of California San Francisco, Dept. of Medicine, Division \\ Cardiology, San Francisco, Calif., USA
}

Background: Intradialytic hypotension and hypertension are both independently associated with mortality among persons with end stage renal disease (ESRD) on hemodialysis. Arterial stiffness and endothelial dysfunction are possible mechanisms for these phenomena, but their associations with hemodynamic instability during dialysis have not been evaluated.

Methods: We recruited 21 patients from SF General and VA chronic dialysis units. Pulse wave velocity (PWV), augmentation index (Aix) of the radial and flow mediated dilation (FMD) of the brachial artery were measured in all except those with severe arrhythmia, non-palpable radial pulse or bilateral upper extremity fistulas. Intradialytic hypotension and hypertension were defined as \% of dialysis sessions over one month associated with a symptomatic decrease in blood pressure $>=20 \mathrm{mmHg}$ or an increase in blood pressure $>=20 \mathrm{mmHg}$ on two or more readings during dialysis. We tested associations of the predictors age, gender, months on dialysis, ultrafiltration, volume overload, pre-dialysis SBP, history of atherosclerotic disease (diagnosis of CAD, CVA or PAD), history of DM, PWV, Aix, and FMD with the outcomes intradialytic hypotension and intradialytic hypertension.

Results: PWV was feasible in 19 subjects (Mean, SD $10.3+/-$ $2.2 \mathrm{~m} / \mathrm{s}$ ); FMD in 16 subjects (Mean, SD $4.3+/-9.1 \%$ ). Intradialytic hypotension or hypertension occurred in more than $1 / 3$ of dialysis sessions for $19 \%$ and $23 \%$ of subjects, respectively. In unadjusted models, each standard deviation decrease in FMD was associated with a $12 \%$ increase in frequency of intradialytic hypotension $(\mathrm{p}=0.01)$. Each standard deviation increase in PWV was associated with a $12 \%$ increase in frequency of intradialytic hypertension $(p<0.001)$. These associations were independent of other predictors.

Conclusion: Endothelial dysfunction may impair coronary flow reserve, leading to low cardiac output and hypotension during dialysis. Arterial stiffness may contribute to intradialytic hypertension, perhaps in concert with high sympathetic tone. We plan larger studies to elucidate these potential mechanisms of hemodynamic instability during dialysis.
28

\section{Dry Weight, Pre-Dialysis Systolic Blood Pressure (SBP) and Intima-Media Thickness (IMT) Predict Pre-Dialysis Brain Natriuretic Peptide (BNP) Levels}

Paolo Lentini', Luca Zanoli, Valentina Pellanda ${ }^{1}$, Vincenzo Catena' ${ }^{2}$, Alexandra Chronopoulos ${ }^{4}$, Massimo De Cal', Claudio Ronco ${ }^{4}$, Marco Baiocchi', Roberto Dell'Aquila'

${ }^{1}$ Nephrology, St. Bassiano Hospital, Bassano Del Grappa (Vi), Italy, 2Intensive Care Unit, St. Bassiano Hospital, Bassano Del Grappa (Vi), Italy, ${ }^{3}$ Internal Medicine, University of Catania, Catania, Italy, ${ }^{4}$ Nephrology, St Bortolo Hospital, Vicenza, Italy

Background: Cardiovascular events (CV) represent the first cause of cardiovascular mortality in chronic hemodialysis (HD). High BNP levels are correlated with cardiovascular damage and poor outcomes in HD patients.

Aim: To determine the independent predictors of vascular damage in a population of chronic HD patients.

Methods: All 74 patients enrolled in our HD program were included. Median pre-dialysis BNP $(183 \mathrm{pg} / \mathrm{mL})$ was used as a cutoff to create two subgroups. Hematological and biochemical variables were obtained by a pre-dialysis blood draw. All patients underwent a doppler of the supra-aortic blood vessels 30-45 days before BNP analysis. All dopplers were executed by the same operator. Student $t$ test and chi-square test were used for comparison. Univariate predictors of BNP were studied in multivariate logistic regression analysis. The model was tested in ROC curve analysis.

Results: Univariate analysis: The high and low pre-dialysis BNP groups were compared, and there was no significant difference in age, time to first dialysis, and levels of calcium, phosphorous and parthormone. Furthermore, patients with BNP $>183$ had significantly lower Hgb, weight and dry weight, higher systolic blood pressure (SBP), pulse pressure, intima-media thickness (IMT), carotid stenosis and $\mathrm{CV}$ events. Logistic regression analysis: Dry-weight $(1 \mathrm{~kg}$ increase of body wieght, OR $0,93,95 \%$ CI $0.88-0.98, \mathrm{p}<0.05$ ), pre-dialysis SBP $(1 \mathrm{mmHg}$ increase, OR $1.03,95 \% \mathrm{CI} 1.01-1.06, \mathrm{p}<0.05)$ and IMT $(0.1 \mathrm{~mm}$ increase, OR $1.21,95 \%$ CI $1.04-1.40, \mathrm{p}<0.05)$ were selected as independent predictors of pre-dialysis BNP $>183$. Hosmerlemeshow Goodness-of-Fit test was not significant $(p=0.264)$, Nagelkerke R-square was 0.33 . The Area under ROC curve for the selected model was 0.78 (95\%CI 0.67-0.88, $\mathrm{p}<0.0001)$.

Conclusion: Dry weight, pre-dialysis SBP and IMT predict higher levels of pre-dialysis BNP. 


\section{9 \\ Use of Heparin-Grafted Hemodialysis Membranes Improves Biocompatibility Performance in Terms of MCP-1 Circulating Level and Oxidative Status}

\author{
Marion Morena', Isabelle Jaussent', Lotfi Chalabi³, \\ Anne-Sophie Bargnoux 4 , Anne-Marie Dupuy", Stéphanie \\ Badiou $^{4}$, Claire Rakic ${ }^{5}$, Michel Thomas ${ }^{5}$, Bernard Canaud 6 , \\ Jean-Paul Cristo/4 \\ ${ }^{1}$ Institut de Recherche et Formation en Dialyse, \\ Montpellier, France, ${ }^{2}$ Inserm U888, Univ Montpellier \\ 1, Montpellier, France, ${ }^{3}$ AIDER, Montpellier, France, \\ ${ }^{4}$ Laboratoire de Biochimie, CHRU, UMR 204 Nutripass, \\ Univ Montpellier 1, Montpellier, France, ${ }^{5}$ Hospal SAS, \\ Lyon, France, ${ }^{6}$ Service de Néphrologie-Hémodialyse \\ et Soins Intensifs, CHRU, UMR 204 Nutripass, Univ \\ Montpellier 1, Montpellier, France
}

Background: This prospective observational study aimed at evaluating efficacy and biocompatibility performances of the new heparin-coated Evodial dialyzers with/without systemic heparin reduction.

Methods: After a four-week wash-out period with reference polysulfone F70S dialyzers, 6 hemodialysis patients were sequentially dialyzed with Evodial, F70S and Evodial dialyzers using 30\% heparin reduction, each period of treatment was four weeks. Removal rates (RR) (urea, creatinine and beta2-microglobulin), dialysis dose and instantaneous clearances (urea and creatinine) were measured as well as inflammatory (CRP, fibrinogen, IL-6, TNF-alpha and MCP-1) and oxidative stress (OS) (superoxide anion, homocysteine and isoprostanes) parameters at the end of each study period.

Results: Patients treated with Evodial or F70S dialyzers for four weeks presented comparable dialysis efficacy parameters including urea and creatinine RR, dialysis dose and instantaneous clearances. By contrast, a significantly lower but reasonably good beta2-microglobulin RR was achieved with Evodial dialyzers. Regarding biocompatibility, no significant difference was observed with inflammation and OS except for postdialysis MCP-1 which significantly decreased with Evodial dialyzers. Thirty percent heparinization reduction with Evodial dialyzers did not induce any change in inflammation but led to an improvement in OS as demonstrated by a decrease in postdialysis superoxide production and predialysis homocysteine and isoprostane.

Conclusion: This bioactive dialyzer together with heparin dose reduction represents a good trade-off between efficacy and biocompatibility performance (improvement in OS with a weak decrease in efficacy) and its use is encouraging for hemodialysis patients not only in reducing OS but also in improving patient comorbid conditions due to lesser heparin side effects.

\section{0}

\section{Circumferential Strain Is Globally Reduced in Incident Haemodialysis Patients Despite Normal Left Ventricular Ejection Fraction: Insights from a Cardiac Magnetic Resonance Tagging Study}

\author{
Aghogho Odudu', Mohamed Tarek Eldehni', Christopher \\ W. Mclntyre ${ }^{2}$ \\ 1Department of Renal Medicine, Royal Derby Hospital, \\ United Kingdom, ${ }^{2}$ School of Graduate Entry Medicine and \\ Health, University of Nottingham, United Kingdom
}

Background: The capability to study myocardial dysfunction in HD patients at a regional level might be of value in developing and monitoring interventions aimed at preventing reduced left ventricular ejection fraction (LVEF) and heart failure. Echocardiographic studies of regional function have been restricted to prevalent HD patients using speckle tracking and doppler strain and are limited by heterogeneity, foreshortened views, preload, angle and operator dependency. Cardiovascular magnetic resonance (CMR) tagging is the reference standard for assessment of regional function and is the most widely validated and reproducible tool for multi-dimensional strain quantification. We report data from the first study to use CMR tagging in HD patients. This novel technique utilises tracking of a magnetized grid to describe and quantify motion of the entire cardiac volume with high spatial and temporal resolution. We studied peak systolic midwall LV circumferential strain as it is recognised as the parameter most suited for assessing the transmural strain gradient.

Methods: 11 incident HD patients (exposed to HD $<90$ days) were studied on a standardised midweek post-HD day using a $1.5 \mathrm{~T}$ MR scanner. Conventional CMR techniques to assess volumes were combined with a $7 \mathrm{~mm}$ grid tag applied with a spatial modulation of magnetization sequence in 3 short axis slices evenly distributed from the LV base to the apex. Harmonic magnitude and phase images were processed and analysed off-line using semi-automated software. Peak systolic circumferential strain (Ecc) was calculated at the mid-wall in short-axis slices at the basal, middle and apical ventricular levels according to the American Heart Association 17 segment model. Values were compared to published control values from CMR tagging of healthy volunteers using the same protocol and analysis.

Results: The mean \pm SD of values are presented. Age $61 \pm 14$ yrs. Global LV function was normal (LVEF $69 \pm 15 \%$ ). There was a high degree of LVH (LVMI $97.7 \pm 25 \mathrm{~m}^{2}$ ). Anterior and lateral segments had greater Ecc than inferior segments consistent with the pattern in controls. However, in all segments Ecc was significantly reduced compared to non-uraemic controls. The data table is presented with strain expressed as a negative percentage by convention at a segmental resolution in the form HD vs Control. BASAL LEVEL: Anterior, $-16 \pm 7$ vs $-22 \pm 2$, Anteroseptal $-12 \pm 4$ vs $-19 \pm 2$, Inferoseptal $-6 \pm 5$ vs $-18 \pm 1$ Inferior $-10 \pm 4$ vs $-16 \pm 2$ Posterolateral, $-13 \pm 8$ vs $-21 \pm 2$ Anterolateral, $-15 \pm 7$ vs $-21 \pm 2$ MID LEVEL: Anterior, $-15 \pm 5$ vs $-23 \pm 2$, Anteroseptal $-13 \pm 4$ vs $-21 \pm 2$, Inferoseptal $-9 \pm 6$ vs $-20 \pm 3$ Inferior $-11 \pm 7$ vs $-18 \pm 2$ Posterolateral, $-9 \pm 5$ vs $-22 \pm 3$ Anterolateral $-13 \pm 6$ vs $-24 \pm 3$ APICAL LEVEL: Anterior, $-20 \pm 10$ vs $-27 \pm 2$ Septal $-14 \pm 6$ vs $-24 \pm 2$ Inferior $-15 \pm 6$ vs $-21 \pm 4$ Lateral $-17 \pm 9$ vs $-25 \pm 3$.

Conclusion: Incident HD patients are not characterised by reduced LVEF more typical of patients established on HD. However study of circumferential strain reveals global reductions in circum- 
ferential strain despite preserved LVEF. The significance of the relationship to HD tolerability and susceptibility to recurrent HD-induced cardiac injury awaits further elucidation.

\section{1 \\ Impact of Low Blood Flow in the Occurrence of Catheter Related Infection}

Fabiana Dias Carneiro, Nadia K Guimaraes-Souza, Henrique Silva dos Santos, Marisa A Souza, Ana Cláudia Mallet, Ilson Jorge lizuka, Adriano Amirati, Maria Claudia Andreolli, Miguel Angelo Goes Júnior, Bento C Santos

Albert Einstein Jewish Hospital, Brazil

Background: We evaluated 33.901 hemodialysis procedures performed in six years at an adult dialysis unit located in an urban tertiary medical center. One fifth of patients had a central venous catheter as a vascular access, and 20 catheter infections occurred in this period. The objective of this study was to analyze the impact of blood flux on the occurrence of catheter infections. Blood flow lower than $200 \mathrm{ml} / \mathrm{min}$ was considered low flux. Catheter infection was diagnosed when hemoculture was positive in at least one catheter lumen and negative in peripheral hemoculture.

Methods: For this work nursing records were analyzed retrospectively. Dialysis procedures, dialysis access, and complications such as: clotting system during dialysis, hypotension, hypertension, cramps where recorded. Hypertension and cramps were analyzed as other complications. Statistical analyzes were done with GraphPad Prism software using T-test and one-way ANOVA.

Results: During 6 years, 33.901 hemodialysis were performed, 26.996 of procedures were in patients with arterious-venous fistula $(79.63 \%)$ and $6.905(20.37 \%)$ with catheters. Twenty catheters infections occurred in this period an average of 3 infections per 1000 procedures. Blood flow lower than $200 \mathrm{ml} / \mathrm{min}$ was associated with catheter infection $(p<0.0001)$, system clotting $(p<0.0001)$. In addition, intradialytic hypotension $(\mathrm{p}<0.0001)$, and others complications $(\mathrm{p}<0.0001)$ were also correlated to blood flow lower than $200 \mathrm{ml} /$ min.

Conclusion: Low blood flow dialysis is associated with catheter infection. In addition, not surprisingly, this retrospective study shows that low flow dialysis is associated with higher incidence of system clotting. Of note, as consequence of patient condition or catheter functionality, intradialytic hypotension and other complications were also more incident in low blood flow dialysis. In conclusion, patients submitted to hemodialysis with low blood flow are in increase risk of complications and higher manipulation of the access leading to increase risk of infection.

\section{Hemodiafiltration}

\section{Vancomycin Dose Adjustment Using Pharmacokinetic Analysis in Critically III Patients Receiving High Volume Continuous Renal Replacement Therapy}

Zahálková J1, Táborský P2, Petejová N³, Urbánek K, Strojil $J^{1}$, Kacírová I' $^{1}$

${ }^{1}$ Faculty of Medicine and University Hospital, Palacky University Olomouc, Czech Republic, ${ }^{2}$ Fresenius Medical

Care, Praha, Czech Republic, ${ }^{3}$ University Hospital, Ostrava, Czech Republic

Background: Current vancomycin (VAN) dosage is targeted to reach higher serum concentration with an optimum AUC24/MIC ration more than 400 . Serum concentration should not decrease below $10 \mathrm{mg} / \mathrm{l}$ to avoid induction of bacterial resistance. High volume continuous renal replacement therapy in patients (pts) with sepsis and acute kidney injury (AKI) is used for metabolic homeostasis control and as an adjuvant therapy of sepsis. This can result in high extracorporeal clearance of administered antibiotics with the risk of underdosage. The aim of the study was to modify the VAN dosage according to the results of predictive pharmacokinetic model, to verify the effectiveness of treatment and provide VAN extracorporeal clearance of high volume continuous venovenous hemofiltration in pre-postdilution mode (Cl-VAN/pre-post HVCVVH).

Methods: Critically ill pts in septic shock and AKI classified according to RIFLE criteria were enrolled in a prospective pilot study. In stage I pts VAN was administered every 12 hours in $1 \mathrm{~h}$. infusion period, in stage F 24-hours intervals were used. Pre-post HVCVVH (50/50) was performed on multiFiltrate FMC using QB $200 \mathrm{ml} / \mathrm{min}$ and Qf $45 \mathrm{ml} / \mathrm{kg}$ b.w./hour. Hemofilters with high-flux polysulfone (PS) membrane were regularly changed every 24 hours. Serum and filtrate VAN concentrations were determined by enzymatic analysis of the device ABBOTT AxSYMTM in the following intervals: before, 1,6 and 12 hour after the VAN administration. PS membrane VAN sieving coefficient (Sc-VAN), Cl-VAN/pre-post HVCVVH and killing characteristic (AUC24:MIC) were determined. Pharmacokinetic analysis was performed on the second day of treatment using software MwPharm 3.30 and following VAN doses and dosing intervals were adjusted.

Results: Preliminary results were obtained in 10 pts (6 pts stage I, 4 pts stage F). Pharmacokinetic analysis resulted in increase of dose in 3 pts stage I and 3 pts stage F. Mean AUC24:MIC was 361 in stage I and 378 in stage F, a targeted AUC24:MIC more than 400 was reached during first 24 hours of treatment in 3 pts of stage I and 4 pts of stage F. Serum VAN conc. before the second dose were insufficient (below $10 \mathrm{mg} / \mathrm{l}$ ) in 4 pts stage I and 2 pts of stage F.In the whole group mean serum VAN conc. measured one hour after first VAN dose was 22,64 (+- 6,44 ) mg/l, after an additional six hours serum VAN conc. declined to $11,85(+-4,69) \mathrm{mg} / \mathrm{l}$, which means reduction by 48 $(+-12) \%$. Clearance VAN/prepost HVCVVH reached 47,40 $(+-9,38)$ $\mathrm{ml} / \mathrm{min}$., Sc-VAN was $0,77(+-0,08)$, diuresis and net ultrafiltration 
rate during first day were $88(+-60) \mathrm{ml} / 24 \mathrm{~h}, 1557(+-792) \mathrm{ml} / 24$ h.resp.

Conclusion: Current recommendations for VAN dosing and monitoring were insufficient for pts with severe sepsis treated by prepost HVCVVH in stage I and partly in stage F of AKI. It is necessary to apply higher first loading dose to ensure sufficient VAN serum concentration during the first day of treatment and to perform serum concentration measurement with computer pharmacokinetic analysis before the second dose. Six hours after the VAN administration decreases its serum concentration on average by half in the ongoing HVCVVH. Selecting this time interval for the first VAN evaluation appears to be helpful to allow sufficient time for estimate the trough VAN concentration.

Supported by grant IGA MZCR NS10309.

\section{Complications}

33

\section{Vascular Calcification Scores on Plain Radiographs of Hands and Pelvis Is Associated with Arterial Stiffness, Inflammation, Nutrition in Hemodialsysis Patients}

Hyung-Jong Kim', Hun Jeong ${ }^{2}$, So-Young Lee ${ }^{1}$, Heo-Young Kim ${ }^{1}$, Dong Ho Yang ${ }^{1}$

${ }^{1}$ Internal Medicine, CHA Bundang Medical Center, CHA University, Seongnam, South Korea, ${ }^{2}$ Internal Medicine, Seoul Bukbu Geriatric Hospital, Seoul, South Korea

Background: Vascular calcification(VC) have been shown to independent predictors of cardiovascular mortality in hemodialysis(HD) patients. Various VC scores have been evaluated in hemodialysis patients using plain radiographic films of the pelvis and hands. The presence of VC in HD patients is associated with increased stiffness of arteries. The purpose of this study was to analysis the association of $\mathrm{VC}$ and arterial stiffness, inflammation, nutrition in HD patients.

Methods: We studied for 101 stable HD patients. All patients underwent both hands and pelvis X-ray. All patients were carried out to measurement of the carotid-femoral pulse wave velocity (PWV), the ankle brachial index (ABI) and augmentation index (AI). The presence of calcifications were analysed as a score (0 to 8$)$ according to the number of arterial sites with calcifications on both hands and pelvis X-ray. Laboratory tests such as albumin, calcium, phosphate, iPTH, C-reactive protein, homocysteine, lipid profile were measured.

Results: Sixty of 101 patients (Diabetes 48 patients) were male. The mean age was $55.7 \pm 13.2$ years and the mean HD duration was $185 \pm 55.8$ months. The study patients were grouped according to VC scores to three groups. Group 1 (61 patients) was not investigation of $\mathrm{VC}$ and group 2 (25 patients) had VC score of 1 to 4 and group 3 (14 patients) had VC score of 4 to 8 . The serum albumin had significant negative correlation with $\mathrm{C}$-reactive protein and VC scores $(\mathrm{p}<0.05)$. The comparisons of different groups showed that $\mathrm{PWV}$ and $\mathrm{ABI}$ of group 3 were significantly increased than group 1 and $2(p<0.05)$. AI was not significant difference among three groups. C-reactive protein and homocysteine were not significant difference among three groups. There was positive correlation between C-reactive protein and PWV $(p<0.05)$ and not correlation between homocysteine and PWV.

Conclusion: The presence of vascular calcifications in HD patients may be associated with increased arterial stiffness, inflammation and nutrition. The plain X-ray of both hands and pelvis is a useful technique associated with arterial stiffness in HD patients. Hence, we thought that VC scores of the pain X-ray of hands and pelvis may be important for estimate of cardiovascular diseases and mortality in HD patients.

\section{4 \\ Does Inflammation Have an Effect on Red Blood Cell Lifespan in Chronic Hemodialysis Patients? \\ Georges Ouellet', Anja Kruse1, Ali Al Sawi', Stephan \\ Thijssen ${ }^{1}$, Viktoriya Kuntsevich², Peter Kotanko', Nathan W. Levin $^{1}$ \\ ${ }^{1}$ Renal Research Institute, New York, NY, ${ }^{2}$ Beth Israel \\ Medical Center, New York, NY, USA}

Background: Although an underappreciated phenomenon, the reduction in red blood cell lifespan (RBCLS) is, along with iron and erythropoietin (EPO) deficiency, a significant contributor to renal anemia and possibly to EPO resistance. We previously reported on the absence of relationship between RBCLS and IL-1beta; IL-6, IL-10, IFN-gamma; and TNF-alpha; in maintenance hemodialysis (HD) patients. In this study, we aimed to further explore the potential effect of inflammation on RBCLS in a group of patients including the 22 previously studied and 11 newly enrolled subjects.

Methods: Non-smoking maintenance HD patients without inflammatory pulmonary condition were recruited. Carbon monoxide $(\mathrm{CO})$ concentration was measured in alveolar and environmental air samples with a 910 Buck Scientific gas chromatograph (Buck Scientific, East Norwalk, CT). RBCLS was computed from endogenous CO production (Strocchi, 1992). EDTA-plasma was aliquoted and frozen at $-70^{\circ} \mathrm{C}$. Quantitative analysis of plasma IL-18 was made by high sensitivity ELISA (MLB, Nagoya, Japan) that uses two monoclonal antibodies against two different epitopes of human IL-18. Plasma levels of IP-10 and TNF-alpha; were analyzed in duplicates using the xMAP technology with 2-plex Human Cytokine/Chemokine MILLIPLEX ${ }^{\mathrm{TM}}$ map Kit on a Luminex $100^{\mathrm{TM}}$ System. Pearson and Spearman correlations were analyzed as appropriate.

Results: Thirty-three patients (64\% males; 70\% Blacks; $48 \%$ diabetics; $13 \%$ catheters; mean age $60.6 \pm 14.5$ years) were enrolled. The median weekly EPO dose was 12600 units (IQR: 5250-20100). The mean RBCLS was $68.7 \pm 22.0$ days, ranging from 33.9 to 122.7 days. No correlation was observed between RBCLS and IL-18 ( $\mathrm{r}=-0.187$, $\mathrm{P}=0.305), \log \mathrm{IP}-10(\mathrm{r}=0.079, \mathrm{P}=0.661)$ and TNF-alpha, $(\mathrm{rho}=-0.009$, $\mathrm{P}=0.960)$.

Conclusion: We found no relationship between RBCLS and inflammatory markers IL-18, IP-10 and TNF- alpha. These findings corroborate our previous observations and suggest that factors other 
than inflammation, such as increased level of uremic toxins or oxidative stress, are responsible for the shortened RBCLS in HD patients.

\section{5 \\ Sevelamer Hydrochloride Can Improve the Patients' Survival in Secondary Hyperparathyroidism (2HPT) Patients Undergoing Maxacalcitol Therapy}

\author{
Takashi Shigematsu, Sigeo Negi \\ Division of Nephrology \& Blood Purification Medicine, \\ Wakayama Medical University, MOST study group, Japan
}

Background: Secondary hyperparathyroidism (2HPT) with hyperphosphatemia and is still major problem in CKD-5D patients. It has been known to be certain risk factor on patients' survival. Positive vitamin D analog treatment is effective therapy on $2 \mathrm{HPT}$, but very often induce hypercalcemia and/or hyperphosphatemia. In Japan, we have treated for 2HPT by Maxacalcitol intravenously as the Japanese original active vitamin D analogue for eight years. However, affecting factors on CKD-5D patients' survival are still obscure undergoing intravenous Maxacalcitol therapy.

Methods: 2HPT patients undergoing Maxacalcitol therapy were enrolled from 25 medical institutes in Maxacalcitol Therapy On SHPT (MOST) study group. We analyzed risk factors on CKD-5D patients' survival with 8 years Maxacalcitol therapy as a multicenter retrospective observational study.

Results: Subjects were 1,303 CKD-5D patients ( $\mathrm{F} / \mathrm{M}=561 / 734$; in 8 patients, the gender was unknown). The mean age was $62.3 \pm 12.69$ years and the mean hemodialysis period was $9.1 \pm 7.4$ years. Subjects were composed of 240 diabetic nephropathy patients, 1059 nondiabetic patients and unclear 4 patients. The mean intact-PTH value for all patients at enrollment was $531.3 \pm 249.6 \mathrm{pg} / \mathrm{ml}$. At the same time, the mean corrected serum Ca was $9.4 \pm 0.9 \mathrm{mg} / \mathrm{dl}$, and the mean serum phosphate was $6.0 \pm 1.2 \mathrm{mg} / \mathrm{dl} .132$ patients were died during the study period. There were 132 deaths with cardiovascular disease (CVD) accounting for one-third of the total mortality. Risk factors that influenced the outcome of death were examined by multivariate Cox regression analysis. The mortality risk was found to increase in association with the diabetic nephropathy (hazard ratio, 2.19), a past history of CVD (hazard ratio, 2.55), and a higher baseline serum phosphate level (hazard ratio, 1.32). In contrast, post-enrollment treatment with sevelamer hydrochloride was associated with a significant reduction of the mortality risk (hazard ratio, $0.22 ; \mathrm{P}<0.001$ ). This risk reduction was not only significant in patients without diabetic nephropathy, but also in patients with diabetic nephropathy. In addition, it was unrelated to either the baseline or post-treatment serum phosphate level.

Conclusion: Our results suggest that sevelamer hydrochloride may be able to reduce the mortality risk in CKD-5D patients with 2HPT patients undergoing positive vitamin D analog treatment.The effect of sevelamer hydrochloride on lipid metabolism and inflammation may play some role to reduce the mortality risk in CKD-5D patients.

\section{6 \\ Plasma Brain Natriuretic Peptide, A Useful Marker of Fluid Overload in Hospitalized Haemodialysis Patients}

\author{
Gulperi Celik, Silinou Emilie, Chazot Charles \\ NephroCare Tassin, France
}

Background: Plasma BNP is synthesized by ventricle cardiomyocytes under stretch constraint. It is frequently increased in haemodialysis (HD) patients because of cardiac disease and also fluid overload. Moreover hospitalization for intercurrent illness frequently disrupts the nutritional status of HD patients and jeopardizes the dry weight prescription. We report in this study the evolution of BNP, blood pressure and body weight in hospitalized HD patients. The goal of the study is to evaluate BNP as a tool for dry weight prescription during intercurrent events.

Methods: We have studied patients who required hospitalization hospitalized during the year 2007. The plasma BNP level was assessed using a chemoluminescent microparticule immunoassay on i8200 Architect (Abbott ${ }^{\mathbb{B}}$, Paris, France; normal value $<100$ pg/ $\mathrm{ml}$ ). The plasma BNP level during hospitalisation (BNP hosp) was compared with the lower value recorded in the patient chart (baseline BNP) and with the lower value in the 4 months following hospital discharge. Pre- and post-dialysis blood pressure and post-dialysis body weight in these different periods were recorded and compared.

Results: Forty-two patients (F/M:18/24; 72.5 y.o.; 19/42 with diabetes) were retained for analysis. The causes for the hospital stay were blood access problems (7\%), cardiac disease (14\%), peripheral artery disease $(19 \%)$, sepsis $(21 \%)$, fractures $(9 \%)$, gastro-intestinal disease $(7 \%)$, cachexia $(7 \%)$, miscellaneous $(16 \%)$. During hospitalization, plasma BNP increased from $421 \mathrm{pg} / \mathrm{ml}$ at baseline to 1584 $\mathrm{pg} / \mathrm{ml}(\mathrm{p}<0.0001$, paired t-test, 40 patients). After hospitalization discharge, BNP decreased from 1223 to $616 \mathrm{pg} / \mathrm{ml}$ ( $\mathrm{p}=0.005$, paired t-test, 24 patients). In these 24 patients, ANOVA for repeated measures found a significant difference between baseline, hospitalisation, and follow-up (respectively at 264, 1223, and $616 \mathrm{pg} / \mathrm{ml}$ ). The post-dialysis BW decreased from 71.0 kilos at baseline to 70.5 during hospitalisation and 67.84 months after discharge. Pre-dialysis blood pressure was not different at baseline and during hospitalization. However post-dialysis systolic BP increased from 104 to $121 \mathrm{mmHg}$ between baseline and the hospitalization stay $(\mathrm{p}=0.05$, paired t-test).

Conclusion: Hence plasma BNP level increases importantly when HD patients present with intercurrent event. It can be interpreted as a marker of fluid overload related to catabolism and inappropriate DW prescription. Decreasing the post-dialysis BW prescription normalizes plasma BNP level that can be used as a reliable marker of extracellular fluid excess during intercurrent illnesses. 
37

\section{Effects of Materials and Pore Sizes of a Various Double Lumen Catheter on Blood Flow Rate}

\author{
Yoshimitsu Takahashi ${ }^{1}$, Isao Tsukamoto ${ }^{2}$, Yoshihisa \\ Yamashita ${ }^{3}$, Hiromichi Suzuki ${ }^{4}$, Hironori Nemoto', Yoshihiko \\ Kanno ${ }^{5}$ \\ ${ }^{1}$ Higasimatsuyama Medical Clinic, ${ }^{2}$ Saitama Medical \\ University International Medical Center Department of \\ Medical Engineering, ${ }^{3}$ Saitama Medical University Faculty \\ of Health and Medical Treatment Biomedical Engineering \\ department, ${ }^{4}$ Saitama Medical University Hospital \\ Department of Nephrology, ${ }^{5}$ Musashi Ranzan Hospital, \\ Japan
}

Background: Double lumen catheters (DLC) are widely used for patients who need continuous renal replacement therapies (CRRT) for obtaining adequate vascular access. In spite of recent improvements in the structure of the catheters, it is uncertain what the materials and pore sizes are adequate at a high blood flow. The aim of this study is to compare actual flow rate of a various type of DLC available in Japan when a high flow rate is applied.

Methods: Experiment 1 . Fifty \% glyceride in solution was circulated at the flow rate of $500 \mathrm{ml} / \mathrm{min}$ in a tube $(10 \mathrm{~mm}$ in the inside diameter) with the tropical aquarium set at $36 \mathrm{C}$. Five different types of DLC connected with circuit with a peristaltic pump were inserted in the tube at the speed of from 100 to $300 \mathrm{ml} / \mathrm{min}$ using a peristaltic pump. During the experiment, fluid flow and pressures were measured and then an actual flow and the predicted flow were compared.

Results: Data obtained from the present study is shown in Table 1. When predicted flow was at rate of $100 \mathrm{ml} / \mathrm{min}$, all DLCs showed minimum differences between the actual and predicted values. However, when predicted flow of faster than $300 \mathrm{ml} / \mathrm{min}$ was applied, Flexxicon II and Gam Gath showed marked differences between the predicted and the actual values compared with other DLCs. Table 1 Increases or decreases (-) of percent changes of the measured values of flow rate from the predicted ones in different double lumen catheters. table 1 .

Conclusion: Present data suggested that when high blood flow are required, the type of DLC will be determined based on their characteristics.

Table 1

\begin{tabular}{llr}
\hline Kind of DLC & $(100 \mathrm{ml} / \mathrm{min}$, & $300 \mathrm{ml} / \mathrm{min})$, \\
\hline Gentle Cath & $(3.4 \pm 1.5$, & $-3.4 \pm 1.2)$, \\
Niagara Slim with side hole & $(2.9 \pm 2.0$, & $-5.5 \pm 1.1)$, \\
Niagara Slim & $(3.1 \pm 1.4$, & $-5.4 \pm 0.5)$, \\
Flexxicon II & $(2.9 \pm 2.0$, & $-11.7 \pm 1.0)$, \\
Gam Cath & $(3.4 \pm 0.8$ & $-7.8 \pm 1.1)$, \\
\hline
\end{tabular}

28th Annual Meeting of the International Society of Blood Purification (ISBP)

\section{8 \\ Development of Hemodialysis Accident Simulator System}

Yoshiaki Nishite', Shingo Takesawa², Heihachi Migita², Yoshihiro Tange? ${ }^{2}$, Vee David ${ }^{2}$

${ }^{1}$ Kinki Univ.Department of Biomedical Engineering,School of Biology-Oriented Science and Technology, Osaka,

${ }^{2}$ Kyushu Univ. of Health and Welfare, Medical Engineering, Nabeoka, Japan

Background: The dialysis device of the hemodialysis treatment uses the operation of the extracorporeal circulation. However, in spite of being the important operation which endangers patient life, in the present situation, the acquisition of the operating technique and trouble handling are except the clinical scene and it becomes impossible to do. An accident example in the dialysis-treatment to think that the operation mistake of the dialysis device and the immaturity of the clinical staffs are cause, too, is reported. Therefore, it tried the development of the simulator which can do simulation training to learn the dealing which is right in case of acquisition of the basic operating technique and the accident in the place except being clinical.

Methods: It paid attention to an alarm sound's (buzzer) and alarm light's (outside indicator light: LED) springing from the dialysis device in the form of the caution occurrence about a wide range of accident and trouble which occurs in the dialysis-treatment. There is equipment which emits light with the LED in the alarm light to tell of the scenery which has the one which emits (by the music) a melody in case of the alarm sound and the one of the buzzer. For the sound and the alarm by the LED to occur at the same time, the staff of dialysistreatment-center can confirm corresponding equipment easily and can do handling. In other words, if it is possible to make an alarm occur from the dialysis device, the accident occurs. A trainee of the training discovers the cause of the accident and makes the simulator which can handle by the appropriate procedure.

Results: It composes a simulator in the following five parts. 1) Suspected blood recirculation circuit part. It installs the bypass circuit to make suspected blood recirculation. By the circulating pump which is installed in the control unit part, it makes suspected blood recirculation. It makes a recirculation flow volume $200 \mathrm{~mL} /$ minute equal to or more than ( the average blood flow volume of Japan ). It makes recirculation arm parts for the simulator and it connects with the bloodcircuits part of the draw blood part and return blood part. 2) Dialysis device and Blood-circuits part. It uses the dialysis device to be using by usual dialysis-treatment at each hospital.Therefore, the attending a lecture can receive a simulation by the status which is the same as the daily work. This part sets proportional Solenoid-controlled valve in each of the draw blood and return blood parts and makes the alarm of the draw blood lack and the rise of the return blood pressure (venouspressure) occur. 3) Dialyzer module part. It sets blood-circuits, and a connection, Solenoid-controlled valve and a filtration filter and it makes the accident of the dialyzer relation occur. It sets Solenoidcontrolled valve and a filtration filter in the dialyzer jacket and it uses as the dialyzer. 4) Control unit part. Because it set in the draw blood 
and the return blood part, Solenoid-controlled valve operates by it and it controls the suspected blood flow volume. The coach operates it from the touchpad. 5) Timer part;It processes an accident after the alarm occurs and it measures the time which returns to the normal condition by the reset. This operates a timer by the optical sensor by setting it on the LED of the alarm light.The hemodialysis simulator system could generate the following alarm with 6 patterns from the above. Arterial pressure decline alarm; Arterial pressure ascending alarm; Venous pressure decline alarm; Venous pressure ascending alarm; Air bubble mixing alarm; Blood leak alarm.

Conclusion: To experience an accident in the dialysis-treatment beforehand clinically and in the educational facilities is important. This system is very useful for the clinical staffs to master the techniques to prevent a number of potential accidents during hemodialysis treatment. Trainees should reach this goal within a short period of time, based on the number of training.In the future, it does simulation training by the simulator and it repeats the verification of the operations check and the simulation of the pilot model and moreover it improves a simulator.

\section{9 \\ Charcoal Filtered Albumin Dialysis Can Reduce Patients Serum Caprylate and Improve Albumin Function and Hemodynamics}

Jan Stange, Sebastian Koball, Sebastian Klammt, Steffen
Mitzner, Michael Hinz, Helga Weiss- Reining, Emil
Reisinger
Medical Clinic II, University of Rostock, Germany

Background: The outcome of Acute on Chronic Liver Failure (AoCLF) is dependent on hemodynamic stability and prevention of secondary organ failure. Liver Support using albumin dialysis aims at removing albumin bound toxins to improve patients albumin capacity $(\mathrm{ABiC})$ to bind vasodilators (e.g. 5-Kynorenine at binding site II) and improve vasodilation and hemodynamic stability. Single pass albumin dialysis (SPAD) using commercial albumin is associated with the risk of transfer of industrial stabilizer caprylate from dialysate albumin into patients blood which might even reduce patients $\mathrm{ABiC}$ since it claims binding site II. Also, Caprylate has been reported to be a vasodilator. The aim was to investigate the effect of removing caprylate from albumin by charcoal filtration prior albumin dialysis on patients caprylate course, patients $\mathrm{ABiC}$ and on hemodynamics.

Methods: In a clinical setting, Norepinephrine (NA) dependent patients with AoCLF were crossed over from SPAD or CVVHD to single pass albumin dialysis with $2 \%$ albumin which was defatted by charcoal filtration. Serum Caprylate concentration, albumin binding capacity $(\mathrm{ABiC})$ at albumins binding site II and hemodynamics were followed.

Results: 4 Patients with AoCLF and anuria presented with a significantly reduced $\mathrm{ABiC}$ to less than $40 \%$ of healthy controls at baseline. Neither CVVHD nor SPAD had a significant effect on patients albumin binding or on patients hemodynamics within the first 24 hours of treatment. SPAD using commercial albumin is associated with significant transfer of caprylate from dialysate into patients blood. When albumin dialysis was applied using charcoal filtered dialysate albu- min it resulted in reduction of caprylate and significant improvement of $\mathrm{ABiC}$ (by more than $10 \%$ per session). In all 4 patients crossing over to charcoal filtered albumin enhanced dialysis was followed by reduction of the need for Norepinephrine and hemodynamic stabilization within 24 hours and recovery of renal function within 5 days.

Conclusion: The observation supports the hypothesis that the function of albumins binding site II, which can be measured by the $\mathrm{ABiC}$ test, plays a role in binding and activating endogenous vasodilators. Albumin contaminations with caprylate inhibit albumin binding at site II and reduce efficacy of albumin dialysis. A prospective randomized Cross Over Trial is planned to confirm the effect of $\mathrm{c}$ of caaprylate free albumin dialysis on patients caprylate level, $\mathrm{ABiC}$ and hemodynamics.

40

\section{Rapid Loss of Clearance in Albumin Dialysis (MARS) That Might Explain Limited Effects in Clinical Trials}

\author{
Jan Stange, Sebastian Koball, Sebastian Klammt, Steffen \\ Mitzner, Helga Weiss-Reining, Emil Reisinger \\ Medical Clinic II, University of Rostock, Germany
}

Background: Albumin dialysis using MARS aims for removal of albumin bound toxins (ABT) to prevent secondary organ failure in Acute on Chronic Liver Failure (AoCLF) and prolong survival. Although a recently published trial investigating the effect on MARS on survival in AoCLF showed a beneficial tendency within the first two weeks it failed significance at 28 days. Besides trial design, there may also be technical limitations of the current technology responsible for the unsatisfying result. Aim: To investigate stability of clearance for ABT during MARS.

Methods: Using a standardized in vitro model using a toxin spiked plasma pool with continuous toxin infusion, the time course of clearance for bilirubin (Bil), bile acids (BA) and the marker sulphobromophtaleine (SBP) was measured over a 6 hour period. In addition, the concentration of industrial albumin stabilizer Caprylate and its effect on Albumin Binding Capacity $(\mathrm{ABiC})$ at patients albumin binding site II was followed.

Results: During a 6 hour treatment, the initial Clearance dropped to $50 \%$ within 90 minutes for BSP, within 75 minutes for BA and within only 40 minutes for Bilirubin. When standard commercial albumin was applied, industrial albumin stabilizer Caprylate entered the patients circuit, limiting the Capacity of MARS to improve ABiC to less than $2 \%$ per treatment session.

Conclusion: Rapid deterioration of the efficacy to remove the target toxins may explain current clinical limitations. More detailed investigation should reveal if the deterioration is due to exhaustion of the adsorption capacity or the membrane transfer of ABT. Also, contamination of industrial albumin with caprylate limits the capacity of MARS to improve patients albumin binding. Further clinical trials should investigate if resolution of such limitations will improve impact of albumin dialysis on survival in AoCLF. 


\section{1}

\section{Stroke Volume Variation (SVV) Monitoring and Acute Kidney Injury (AKI) in Abdominal Aortic Aneurysm (AAA) Surgery}

\author{
Paolo Lentini ${ }^{1}$, Vincenzo Catena ${ }^{2}$, Rudi Stramanà ${ }^{3}$, \\ Valentina Pellanda ${ }^{1}$, Alexandra Chronopoulos ${ }^{4}$, Massimo \\ De Cal', Claudio Ronco ${ }^{4}$, Marco Baiocchi², Diego \\ Cognolato ${ }^{3}$, Roberto Dell'Aquila' \\ ${ }^{1}$ Nephrology, S. Bassiano Hospital, Bassano Del Grappa \\ (VI), Italy, ${ }^{2}$ Intensive Care Unit, S. Bassiano Hospital, \\ Bassano Del Grappa (VI), Italy, ${ }^{3}$ Vascular Surgery, \\ St. Bassiano, Bassano Del Grappa (VI), Italy, ${ }^{4}$ Nephrology, \\ St. Bortolo Hospital, Vicenza, Italy
}

Background: Patients who undergo surgical interventions of $\mathrm{AAA}$ are at higher risk for acute kidney injury (AKI) development than other surgical patients. AKI can complicate the post-operative course by increasing length of stay and mortality. AKI is often due to renal ischemia, and several mechanisms may be involved such as hypotension and hypoperfusion due to hypovolemia, haemorrhage, decreased cardiac output, vascular disruption, thrombo-embolic phenomena, and inflammation. SVV is a dynamic cardiac preload parameter, and its wide variations are described in the literature as a predictor of volume responsiveness in several populations of mechanically ventilated. We conducted a pilot study to assess if patients with wide variations of SVV before and after clamping of the aorta are at higher risk of AKI development than those with lesser variations.

Methods: The study protocol was approved by our ethics committee. We enrolled 8 consecutive patients undergoing elective AAA surgery with supra-renal clamping. Patients were all mechanically ventilated and fully controlled with volume-control mechanical ventilation, tidal volumes of $8 \mathrm{ml}$ per $\mathrm{kg}$ of predicted body weight, inspiratory:expiratory ratio of $1: 2$, and positive end expiratory pressure of $4 \mathrm{cmH} 20$. Patients were excluded if their respirations were not entirely controlled, if they developed sustained arrhythmias, or if they were extubated before 12 hours post-operative. In all patients SVV was measured with the FlowTrack/Vigileo device by experienced anaesthesiologists and intensivists immediately after endotracheal intubation and after aortic clamp time, every 3 minutes during the procedure, immediately after declamp time and surgical closure, and 24 hours after surgery. A full set of vital signs and ventilator parameters were also noted at those times, and blood was drawn for basic chemistry, renal function, complete blood count, blood gas, and BNP. A detailed log of ingested and excreted fluids was kept for each patient.All patients were hydrated with $70 \mathrm{ml} / \mathrm{kg} / \mathrm{h}$ of crystalloids, and fluid boluses and transfusions were given if needed according to treating medical team preference. Data was compared with nonparametric tests.

Results: Out of 8 patients, 3 developed AKI, defined as RIFLE Risk category, in the first 12 post-operative hours. The serum creatinine returned to normal in the $24-48$ hours following surgery in 3 patients, whereas it remained elevated for one week in the 4th patient. As compared to patients without AKI, AKI patients had a significantly larger SVV after aortic clamping (13.25 vs. $24.5, \mathrm{p}=0.01)$. The increase in SVV at aortic declamping time, as compared to SSV at clamping time, was also significantly higher in AKI patients (-3.75 vs. $12.5, \mathrm{p}=0.04)$.

28th Annual Meeting of the International Society of Blood Purification (ISBP)
Conclusion: SVV monitoring during and after suprarenal AAA surgery is a feasible non-invasive tool that can help identify patients at risk for AKI development. Particularly, an increase in SVV at aortic declamping time, as compared to SSV at clamping time, may be a marker for risk of AKI development that should be further studied.

\section{Apheresis}

\section{2 \\ Catastrophic Antiphospholipid Syndrome and Preganancy}

\begin{abstract}
Amelia Bernasconi', Liliana Voto², Rosa Waisman², Marcela Canteli ${ }^{1}$, Ricardo Heguilen ${ }^{1}$

${ }^{1}$ Division of Nephrology, Hospital Juan A. Fernandez, Buenos Aires, Argentina, ${ }^{2}$ Division of Obstetrics and Gynecology, Hospital Juan A. Fernandez, Buenos Aires, Argentina
\end{abstract}

Background: Although women with antiphospholipid antibodies (APS) have an unusually high proportion of pregnancy losses within the fetal period and adverse outcomes have been reported; pregnancy is in some cases, successful. The aim of this retrospective analysis was to describe the maternal and perinatal outcome of patients with APS.

Methods: We describe the results of 3 pregnant women suffering from APS, and highlight the myriad behaviour of this sometime devastating condition.

Results: Two of them had had a previous uneventful pregnancy 10 years ago, no one displayed a history of thrombosis, 1/3 was associated with systemic lupus erythematosus, and $3 / 3$ had recurrent pregnancy loss. All showed reactive anticardiolipin antibodies, 1/3 lupus anticoagulant, and 1/3 both of them. Increased blood pressure was the most common complication. Two of them received labetalol and amlodipine while the other was treated with alfa methyl dopa. Proteinuria was present in $2 / 3$ pt. Renal function was impaired in two of them, only one requiered haemodialysis (HD). 1/3 pt delivered by programed cesarean section. One pt developed catastrophic APS one week postpartum, with deep vein trombosis, progressive renal failure and was treated with high dose prednisolone, plasma exchange (PE) and HD. As she didn't recover renal function a percutaneous renal biopsy was performed one month later, demonstrating findings of severe thrombotic microangiopathy along with severe tubular atrophy and interstitial fibrosis. Unfortunately the same day she developed cerebral thrombosis. There were no maternal deaths. All women began treatment from the first trimester. They received heparin and low dose aspirin, $1 \mathrm{pt}$ heparin, low dose aspirin and prednisone, hydrochloroquine and azathioprine as she developed a SLE flare, and the remaining case was treated with PE and HD. All cases gave birth to a live newborn. Intrauterine growth restriction was present in $3 / 3$.

Conclusion: Early treatment combined with close maternalfetal surveillance was associated with a chance of live birth rate. Since thrombosis tends to be a self-perpetuating process, an aggres- 
sive therapeutic and multidisciplinary approach is needed but prematurity, preeclampsia and intrauterine growth restriction are common.

43

\section{CRP as a Therapeutic Target: Cardiac Infarction Therapy in Pigs}

Rudolf Kunze ${ }^{3}$, Ahmed Sheriff', Birgit Vogt ${ }^{1}$, Martin Möckel', Ralf Schindler ${ }^{1}$

${ }^{1}$ Dept. Nephrology, Campus Virchow, Charité Universitätsmedizin, Berlin-Wedding, Germany, ${ }^{2}$ Cardiology and Intensive Care, Campus Virchow, Charité Universitätsmedizin, Berlin-Wedding, Germany, ${ }^{3}$ Office Campus Max Delbrueck Centrum, Berlin-Buch, Germany

Background: The pentraxin CRP is an acute phase protein and a marker for infection or AMI. It was suggested that an increase in its blood concentration is a prognostic marker for the risk of cardiac events. Evidences accumulated that CRP is responsible for an extension of the area of infarct in AMI. It can be assumed that immunological processes enhance the destruction of involved heart areas via the CRP-complement pathway in a notable part of the affected patients. CRP binds to lysophosphatidylcholine which is accessible in the membrane of necrotic and late apoptotic cells. Although this function is vitally important for the efficient clearance of materials from destroyed cells, extended tissue destruction caused by high and especially long lasting levels of CRP is contra productive, prolongs healing processes and enhances remodelling. Therefore, CRP removal from blood may be useful for acute treatment of patients with AMI.

Methods: Our therapeutic approach is to lower the CRP concentration in blood after AMI by selective apheresis. We adapted a porcine infarction model (balloon catheter; $n=2 \times 5$ ). A regenerative CRP-specific adsorbing system was created for the depletion of CRP from plasma. After cell-plasma separation by filtering, the plasma was managed by a pump system (ADAsorb) with a specifically adapted software program. The first treatment started at about 16 hours after AMI, the second one after 44 hours. The treatment lasted for 2-3 hours. Two plasma volumes were treated. The CRP plasma concentration was reduced by $60-70 \%$. We established a human like clinical care situation including magnetic resonance imaging. The observation period after infarction was 14 days. Infarct area - estimated by different methods - was the primary clinical endpoint.

Results: We observed a significant decrease in infarct area (by MRI and histology of heart slices) in the verum (CRP apheresis) group but not in the control group (extracorporal plasma managing in a bypass system). In addition, we preserved nearly $60 \%$ of the ejection fraction in the verum group while decreased to about $45 \%$ in the control group $(\mathrm{p}=0.016)$.

Conclusion: The results indicate an important, probably causative role of CRP for the progress and outcome of myocardial infarction. The data confirm our hypothesis about the pathological relevance of high levels of CRP in AMI and demonstrated the clinical benefit of CRP removal from blood. 


\section{Author Index}

Numbers refer to abstract numbers

Al Sawi, A. 34

Algranati, S. 7, 13

Amani, E. 11

Amirati, A. 31

Ammirati, A.L. 24

Ananthapanyasut, W. 11

Andreolli, M.C. 31

Baccarin, M. 5

Badiou, S. 29

Baiocchi, M. 5, 28, 41

Bargnoux, A.-S. 29

Bedini Rocca, M. 7

Bernabeu, R. 6

Bernasconi, A. 42

Bibiloni, M. 6

Blake, D.R. 23

Bourdeau, J.E. 2

Broyles, J. 21

Campese, V. 11

Canada, R. 21

Canaud, B. 29

Canteli, M. 42

Cardoso, R.M. 24

Carneiro, F.D. 24, 31

Castillo, M. 6

Catena, V. 5, 28, 41

Chalabi, L. 29

Chazot, C. 36

Cheng, X. 10

Chertow, G.M. 17

Chronopoulos, A. 5, 28, 41

Claure-Del Granado, R. 17

Cognolato, D. 41

Cristol, J.-P. 29

Crucelegui, S. 7, 13

Cruz Andreoli, M.C. 24

da Carvalho Matos, A.C. 24

David, V. 8, 38

De Cal, M. 5, 28, 41

Dell'Aquila, R. 5, 28, 41

dos Santos, B.F.C. 24

dos Santos, H.S. 31

Dubin, R. 27

Dupuy, A.-M. 29

Eduardo, S.R. 7, 13

Eldehni, M.T. 30

Fortuny, R. 6

Francisco, L. 25

Fu, J. 2

Fu, P. 10

Gadano, A. 7
Gascó, J. 6

Ghaffari, A. 11

Giannasi, S. 7, 13

Goes Junior, M.A. 24, 31

Gold, R.M. 2

Golomb, J. 26

Goto, H. 9

Greloni, G. 7, 13

Guillermo, R.D. 7, 13

Guimaraes-Souza, N.K 31

Gulperi, C. 36

Hamamoto, N. 16

Heguilen, R. 42

Himmelfarb, J. 17

Hinz, M. 15, 39

Hu, S.-E. 18,19

Hudson, J. 21

Ide, T. 16

Iizuka, I.J. 24, 31

Ikizler, A. 17

Iñigo, V. 6

Isakozawa, Y. 8, 14

Ito, A. 20

Izumi, Y. 9

Jaussent, I. 29

Jeong, H. 33

Johansen, K. 27

Júnior, M.A.G. 31

Kacírová, I. 32

Kanno, Y. 37

Kaplan, A.A. 1

Karan, C. 24

Kato, F. 20

Kato, S. 20

Kim, H. 18, 19

Kim, H.-J. 33

Kim, H.-Y. 33

Klammt, S. 39, 40

Koball, S. 15, 39, 40

Kotanko, P. 34

Kruse, A. 34

Kunihiro, C. 12

Kuntsevich, V. 34

Kunze, R. 43

Lee, H.J. 23

Lee, K.-B. 18, 19

Lee, S.-Y. 33

Lentini, P. 5, 28, 41

Levin, N.W. 34

Levine, R. 25

Li, Z. 10

Lynn, R.I. 25
Macedo, E. 17

McIntyre, C.W. 30

Makio, K. 16

Mallet Ramos, A.C. 24,31

Maruyama, S. 20

Masaaki, N. 12

Masazumi, S. 12

Mascarós, V. 6

Mehta, R.L. 17

Migita, H. 14, 38

Minakata, S. 16

Mitzner, S. 15, 39, 40

Miyazi, H. 16

Möckel, M. 43

Moradi, H. 22

Morena, M. 29

Morimoto, S. 16

Muro, H. 16

Nagake, Y. 14

Nakagawa, I. 9

Nakamura, F. 9

Naramura, T. 16

Negi, S. 35

Nemoto, H. 37

Nishite, Y. 14, 38

Nissenson, A.R. 26

Numata, S. 16

Odudu, A. 30

Ouellet, G. 34

Owens, C. 27

Paganini, E.P. 17

Pahl, M.V. 23

Park, J.-Y. 18, 19

Pellanda, V. 5, 28, 41

Peralta, C.A. 27

Petejová, N. 32

Pinner, N. 21

Provenzano, R. 25

Raju, S. 11

Rakic, C. 29

Ramos, A.C.M. 24

Rangel, E. 24

Reisinger, E. 39, 40

Rizvi, A. 11

Ronco, C. 5, 28, 41

Said, H. 22

Sangen, H. 16

San Roman, E. 7, 13

Sano, H. 16

Santos, B.C. 31

Schiller, N. 27

Schindler, R. 43
Serra, D. 7

Servera, M. 6

Sheriff, A. 43

Shigematsu, T. 35

Silinou, E. 36

Simon, S. 25

Soroko, S. 17

Souza, M.A 31

Spach, K. 25

Stange, J. 15, 39, 40

Stramanà, R. 41

Strojil, J. 32

Suzuki, H. 37

Táborský, P. 32

Tadayuki, K. 12

Takahashi, Y. 37

Takai, N. 20

Takashi, S. 12

Takesawa, S. 8, 14, 38

Tange, Y. 8, 14, 38

Teerlink, J. 27

Thijssen, S. 34

Thomas, M. 29

Tsukamoto, I. 37

Tsuruta, Y. 20

Tuttle, A. 1

Udupa, A.D. 2

Ueno, M. 16

Urbánek, K. 32

Varela, F. 7, 13

Vaziri, N. 22

Vaziri, N.D. 23

Vogt, B. 43

Volz, A. 25

von Hartitzsch, B. 2, 3, 4

Voto, L. 42

Waisman, R. 42

Weiss-Reining, H. 39, 40

Weldon, J. 26

Wilson, S. 26

Yamashita, Y. 37

Yang, D.H. 33

Yang, L. 10

Yasuda, Y. 20

Yoshitake, S. 14

Yuzawa, Y. 20

Zahálková, J. 32

Zanoli, L. 5, 28

\section{KARGER}

(C) 2010 S. Karger AG, Basel

Fax +4161306 1234

E-Mail karger@karger.ch

www.karger.com

Accessible online at:

www.karger.com/bpu 